\title{
Algebraic Study on the Super-KP Hierarchy and the Ortho-Symplectic Super-KP Hierarchy
}

\author{
Kimio Uneo ${ }^{1}$, Hirofumi Yamada ${ }^{2}$ and Kaoru Ikeda ${ }^{2}$ \\ ${ }^{1}$ Department of Mathematics, Waseda University, 3-4-1 Ohkubo, Shinjuku-Ku, Tokyo 160, Japan \\ ${ }^{2}$ Department of Mathematics, Tokyo Metropolitan University, 2-1-1 Fukasawa, Setagaya-Ku, Tokyo \\ 158, Japan
}

\begin{abstract}
Bilinear residue formulas are established for the super-KP hierarchy and the ortho-symplectic super-KP hierarchy. Furthermore, superframes corresponding to the ortho-symplectic super-KP hierarchy are completely characterized. Soliton solutions to the super-KP hierarchy are given.
\end{abstract}

\section{Introduction}

This paper is devoted to algebraic study of super-wave functions and soliton solutions of the super Kadomtsev-Petviashvili (SKP) hierarchy and the orthosymplectic (OSp) SKP hierarchy.

The SKP hierarchy was first introduced by Manin-Rudal [12] and was extensively studied by Ueno-Yamada [17-20], Yamada [21], Mulase [13], Ikeda [9] and Radul [14]. Especially, in [19] we proved that the SKP hierarchy equivalently leads to the super-Grassmann equation that connects a point in the universal super-Grassmann manifold $U S G M$ with an initial data of a solution. In that argument, the Birkhoff (Riemann-Hilbert) decomposition in the group of super-microdifferential operators plays a key role. However this operator formalism is rather inconvenient for treating geometrical solutions such as soliton solutions and super-quasi-periodic solutions. We therefore require a super-wave function, as in the case of the ordinary soliton theory.

The theory of the KP hierarchy itself is explained as follows $[2,6,15,16]$ : Let $\mathscr{R}$ be the ring of formal power series over $\mathbf{C}, \mathscr{R}=\mathbf{C}[[x, t]]$ ( $x$ is a space variable and $t=\left(t_{1}, t_{2}, t_{3}, \ldots\right)$ an infinite number of time variables.). The algebra $\mathscr{R}$ is a differential algebra with a derivation $\partial_{x}=\partial / \partial x$. By $\mathscr{E}_{\mathscr{R}}$ we denote the ring of microdifferential operators over $\mathscr{R}$,

$$
\mathscr{E}_{\mathscr{R}}=\mathscr{R}\left(\left(\partial_{x}{ }^{-1}\right)\right)=\left\{\sum_{-\infty<v \ll+\infty} p_{v}(x, t) \partial_{x}{ }^{v} \mid p_{v}(x, t) \in \mathscr{R}\right\} .
$$

A wave operator

$$
W=W\left(x, t, \partial_{x}\right)=\sum_{j=0}^{\infty} w_{j}(x, t) \partial_{x}^{-j} \quad\left(w_{0}=1\right)
$$


is a monic element in $\mathscr{E}_{\mathscr{R}}$ of 0 -th order satisfying the Sato equations

$$
\frac{\partial W}{\partial t_{n}}=B_{n} W-W \partial_{x}{ }^{n}
$$

where $B_{n}=\left(W \partial_{x}{ }^{n} W^{-1}\right)_{+}\left(=\right.$the differential operator part of $\left.W \partial_{x}{ }^{n} W^{-1}\right)$. The compatibility conditions for (1.2) give rise to the Lax or the Zakharov-Shabat representations of the KP hierarchy. A wave function and its dual version are introduced by

$$
\begin{gathered}
w(x, t, \lambda)=W\left(x, t, \partial_{x}\right)\left(\exp \left(x \lambda+\sum_{n=1}^{\infty} t_{n} \lambda^{n}\right)\right), \\
w^{*}(x, t, \lambda)=\left(W^{*}\left(x, t, \partial_{x}\right)\right)^{-1}\left(\exp \left(-x \lambda-\sum_{n=1}^{\infty} t_{n} \lambda^{n}\right)\right),
\end{gathered}
$$

where $W^{*}=\sum_{j=0}^{\infty}\left(-\partial_{x}\right)^{-j} w_{j}(x, t)$ is the formal adjoint operator of $W$. (In general, for $P \in \mathscr{E}_{\mathscr{R}}, P^{*}$ stands for the formal adjoint operator of $P$.) A wave function for the KP hierarchy and its dual are completely characterized by the following bilinear residue formula (BRF):

$$
\operatorname{Res}_{\lambda=\infty}\left(d \lambda w(x, t, \lambda) w^{*}\left(x^{\prime}, t^{\prime}, \lambda\right)\right)=0 .
$$

This BRF is obtained through consideration on the duality of the Laplace transform [a primitive communication with $\mathrm{M}$. Noumi]. In the definition of the BKP and CKP hierarchies [3-5], the even time evolutions are suppressed. Hence $t=\left(t_{1}, t_{3}, \ldots\right)$. We further impose some additional conditions on a wave operator:

$$
\begin{aligned}
& \text { (BKP) } W^{-1}=\partial_{x}{ }^{-1} W^{*} \partial_{x}, \\
& \text { (CKP) } W^{-1}=W^{*} .
\end{aligned}
$$

The BRF for these hierarchies are as follows:

$$
\begin{gathered}
\text { (BKP) } \operatorname{Res}_{\lambda=\infty}\left(d \lambda / \lambda w(x, t, \lambda) w\left(x^{\prime}, t^{\prime},-\lambda\right)\right)=1, \\
(\text { CKP }) \operatorname{Res}_{\lambda=\infty}\left(d \lambda w(x, t, \lambda) w\left(x^{\prime}, t^{\prime},-\lambda\right)\right)=0 .
\end{gathered}
$$

A supersymmetric extension of differential calculus on $\mathscr{R}$ are accomplished by replacing $\partial_{x}$ by $D=\partial_{\theta}+\theta \partial_{x}$, where $\theta$ is an abstract Grassmann variable; $\theta^{2}=0$. The operator $D$ is a square root of $\partial_{x}$.

The SKP hierarchy is described by the Sato equations:

$$
D_{n}(W)=\varepsilon_{n}\left(B_{n} W-W D^{n}\right), \quad B_{n}=\left(W D^{n} W^{-1}\right)_{+}, \quad n=1,2,3, \ldots,
$$

where $W=\sum_{j=0}^{\infty} w_{j}(x, \theta, t) D^{-j}$ is a monic super-microdifferential operator (a superwave operator), $D_{n}$ are super-vector fields with the parity $\underline{n}$ and $\varepsilon_{n}=(-)^{n(n+1) / 2}$. (For the precise definition, see Sect. 2.) The main results in [19] are that the SKP hierarchy can be interpreted as a dynamical system on USGM, the Lie superalgebra $\mathfrak{g l}(\infty \mid \infty)$ appears as the infinitesimal transformation group on the solution space of the SKP hierarchy. As for the super-Fock representation of $\mathfrak{g l}(\infty \mid \infty)$, see [1.10]. 
Using the so-called "2-spinor representation" of super-microdifferential operators, we furthermore show that there is a natural projection map from the solution space of the SKP hierarchy to the direct product of two copies of the solution space of the KP hierarchy.

We define a super-wave function associated with a super-wave operator $W$ by

$$
w(x, \theta, t, \lambda, \xi)=W(x, \theta, t, D)(\exp H(x, \theta, t, \lambda, \xi))
$$

with an appropriate phase factor $H(x, \theta, t, \lambda, \xi)$. where $(\lambda, \xi)$ are (1|1)-dimensional spectral parameters ( $\lambda$ is even, $\xi$ is odd). One of the main results in this paper is the characterization of a super-wave function and its dual of the SKP hierarchy by the following BRF:

$$
\operatorname{Res}_{\lambda=\infty}\left(\Delta(d \lambda / d \xi) w(x, \theta, t, \lambda, \xi) w^{*}\left(x^{\prime}, \theta^{\prime}, t^{\prime}, \lambda, \xi\right)\right)=0,
$$

where $\Delta(d \lambda / d \xi)$ is the super-volume form on the $(\lambda, \xi)$-space (odd quantity). To show this BRF, we establish the theory of the super-Laplace transform and its duality.

By adding a symmetry condition for a super-wave operator of the SKP hierarchy, one obtains the OSp-SKP hierarchy which is related with the infinite dimensional Lie superalgebra $\mathfrak{o s p}(\infty \mid \infty)$. As in the case of the SKP hierarchy, there is a projection map from the OSp-SKP hierarchy to the direct product of the BKP and the CKP hierarchies. The BRF for the OSp-SKP hierarchy is also obtained.

This paper is organized as follows. Section 2 outlines the theory of the SKP hierarchy [19], including some new results: We establish the one-to-one correspondence between formally regular solutions to the hierarchy and points in the biggest cell of USGM. Furthermore, we describe the hierarchy in the 2-spinor picture. In Sect. 3, we will introduce a super-wave function and its dual for the SKP hierarchy. Through analysis of the super-Laplace transform, we prove the BRF for super-microdifferential operators (Theorem 3.6), and for a super-wave function and its dual (Theorem 3.7). Section 4 is devoted to a study of the OSp-SKP hierarchy, especially the BRF (Theorem 4.1). We also give a characterization of the OSp-SKP hierarchy by superframes in the biggest cell of USGM (Theorem 4.6). In Sect. 5, we construct soliton solutions to the SKP hierarchy by means of the so-called direct method.

\section{The SKP Hierarchy and the Universal Super-Grassmann Manifold}

In this section we review the theory of the super-KP hierarchy developed in [17-19]. We will omit proofs of the propositions except for Proposition 2.3 and Proposition 2.5. For the details, see [19].

Let $\mathscr{A}$ be a Grassmann algebra of finite or infinite dimensions over $\mathbf{C}$, and $t=\left(t_{1}, t_{2}, \ldots\right)$ super-time variables $\left(t_{2 k}\right.$ are even, $t_{2 k-1}$ are odd). The supercommutative algebra $\mathscr{S}$ of superfields is, by definition

$$
\mathscr{S}=\mathbf{C}[[x, \theta, t]] \otimes \mathscr{A} .
$$

We introduce naturally the $\mathbf{Z}_{2}$-gradation of $\mathscr{S}, \mathscr{S}=\mathscr{S}_{0} \oplus \mathscr{S}_{1}$ and define the body 
map $\varepsilon$ by the canonical projection

$$
\varepsilon: \mathscr{S} \rightarrow \mathscr{R}=\mathscr{S} /\left(\mathscr{S}_{1}\right)=\mathbf{C}\left[\left[x, t_{2}, t_{4}, \ldots\right]\right],
$$

where $\left(\mathscr{S}_{1}\right)$ is the ideal generated by the subspace $\mathscr{S}_{1}$. A super-differential operator $D=(\partial / \partial \theta)+\theta(\partial / \partial x)$ and super-vector fields

$$
D_{2 l}=\frac{\partial}{\partial t_{2 l}}, \quad D_{2 l-1}=\frac{\partial}{\partial t_{2 l-1}}+\sum_{k=1}^{\infty} t_{2 k-1} \frac{\partial}{\partial t_{2 l+2 k-2}}
$$

act on $\mathscr{S}$. They satisfy the following commutation and anti-commutation relations [12]:

$$
\begin{aligned}
{\left[D, D_{l}\right]_{(-)^{l-1}} } & =0, \quad\left[D_{2 l}, D_{2 k}\right]=\left[D_{2 l}, D_{2 k-1}\right]=0, \\
{\left[D_{2 l-1}, D_{2 k-1}\right]_{+} } & =2 D_{2 l+2 k-2} .
\end{aligned}
$$

We define the algebra $\mathscr{D}$ of super-differential operators by $\mathscr{D}=\mathscr{S}[D]$. Adding the formal inverse element $D^{-1}=\theta+(\partial / \partial \theta)(\partial / \partial x)^{-1}$ to $\mathscr{D}$, we obtain the algebra of super-microdifferential operators. Precisely,

$$
\mathscr{E}=\mathbf{C}[[x, \theta, t]]\left(\left(D^{-1}\right)\right) \otimes \mathscr{A} .
$$

The algebra structure of $\mathscr{E}$ is prescribed by the generalized super-Leibniz rule [12]:

$$
\begin{aligned}
D^{2 k} \cdot f & =\sum_{j=0}^{\infty}\left(\begin{array}{l}
k \\
j
\end{array}\right) D^{2 j}(f) D^{2 k-2 j}, \\
D^{2 k+1} \cdot f & =\sum_{j=0}^{\infty}\left(\begin{array}{l}
k \\
j
\end{array}\right) D^{2 j+1}(f) D^{2 k-2 j}+(-)^{a} \sum_{j=0}^{\infty}\left(\begin{array}{l}
k \\
j
\end{array}\right) 2^{2 j}(f) D^{2 k-2 j+1},
\end{aligned}
$$

for any integer $k$ and $f \in \mathscr{S}_{\underline{a}}$. The algebra $\mathscr{E}$ is endowed with a natural $\mathbf{Z}_{2}$-gradation, $\mathscr{E}=\mathscr{E}_{\underline{0}} \oplus \mathscr{E}_{\underline{1}}$. Namely an operator $P=\sum_{-\infty<j \ll \infty} p_{j}(x, \theta, t) D^{j} \in \mathscr{E}_{\underline{a}}(a=0,1)$ if and only if $p_{j}(x, \theta, t) \in \mathscr{S}_{a+j}$ for any $j$. Moreover we define the body part $\varepsilon(P)$ (we use the same notation as the body map on $\mathscr{S}$ ) by

$$
\varepsilon(P)=\sum_{j: \text { even }} \varepsilon\left(p_{j}(x, \theta, t)\right) \partial_{x}^{j / 2},
$$

which is a microdifferential operator with coefficients in $\mathscr{R}$.

Now we introduce the SKP hierarchy $[12,17-19]$. Let $L$ be a super-microdifferential operator

$$
L=\sum_{i=0}^{\infty} u_{i} D^{1-i} \in \mathscr{E}_{1}
$$

with $u_{0}=1, D\left(u_{1}\right)+2 u_{2}=0$. The SKP hierarchy is a system of the Lax equations:

$$
\begin{aligned}
D_{2 l}(L) & =(-)^{l}\left[\mathrm{~B}_{2 l}, L\right], \\
D_{2 l-1}(L) & =(-)^{l}\left\{\left[B_{2 l-1}, L\right]_{+}-2 L^{2 l}\right\}, \quad l=1,2, \ldots,
\end{aligned}
$$

where $B_{l}=\left(L^{l}\right)_{+} \quad\left(=\right.$ the super-differential operator part of $\left.L^{l}\right)$, and $D_{l}(L)=$ $\Sigma D_{l}\left(u_{i}\right) D^{1-i}$. The system (2.1) is equivalent to a system of the Zakharov-Shabat 
equations:

$$
\begin{aligned}
(-)^{k} D_{2 k}\left(B_{2 l}\right)-(-)^{l} D_{2 l}\left(B_{2 k}\right)+\left[B_{2 l}, B_{2 k}\right] & =0, \\
(-)^{k} D_{2 k}\left(B_{2 l-1}\right)-(-)^{l} D_{2 l-1}\left(B_{2 k}\right)+\left[B_{2 l-1}, B_{2 k}\right] & =0, \\
(-)^{k} D_{2 k-1}\left(B_{2 l-1}\right)+(-)^{l} D_{2 l-1}\left(B_{2 k-1}\right)-\left[B_{2 l-1}, B_{2 k-1}\right]_{+}+2 B_{2 l+2 k-2} & =0, \\
k, l=1,2, \ldots &
\end{aligned}
$$

The first equation in (2.2) with $k=2, l=3$ gives rise to the SKP equation, which is regarded as a supersymmetric extension of the single KP equation: Set

$$
\begin{aligned}
& B_{4}=D^{4}+2 v_{3} D+2 v_{4}, \\
& B_{6}=D^{6}+3 v_{3} D^{3}+3 v_{4} D^{2}+v_{5} D+v_{6} \quad\left(v_{j} \in \mathscr{S}_{\underline{J}}\right) .
\end{aligned}
$$

Then the SKP equation reads

$$
\begin{aligned}
3 D_{4}\left(v_{3}\right) & =-3 v_{3, x x}+2 v_{5, x}, \\
3 D_{4}\left(v_{4}\right) & =-3 v_{4, x x}+6 v_{3} v_{3, x}-4 v_{3} v_{5}+2 v_{6, x}, \\
D_{4}\left(v_{5}\right)+D_{6}\left(v_{3}\right) & =v_{5, x x}-2 v_{3, x x x}-6 v_{3} D\left(v_{3, x}\right)-6\left(v_{3} v_{4}\right)_{x}-2 D\left(v_{3} v_{5}\right), \\
D_{4}\left(v_{6}\right)+D_{6}\left(v_{4}\right) & =v_{6, x x}+2 v_{3} D\left(v_{6}\right)-2 v_{4, x x x}-6 v_{3} D\left(v_{4, x}\right)-6 v_{4} v_{4, x}+2 D\left(v_{4}\right) v_{5} .
\end{aligned}
$$

Before describing the procedure of integrating the SKP hierarchy, we consider a matrix representation of the algebra $\mathscr{E}$. Let

$$
\psi: \mathscr{E} \rightarrow \operatorname{Mat}(\mathbf{Z} ; \mathscr{S})
$$

be an algebra homomorphism defined by $\psi(P)=\left(\psi(P)_{\mu \nu}\right)_{\mu, v \in \mathbf{Z}}\left(P \in \mathscr{E}, \psi(P)_{\mu v} \in \mathscr{S}\right)$ with the matrix entries prescribed by

$$
D^{\mu} \cdot P=\sum_{j \in \mathbf{Z}} \psi(P)_{\mu v} D^{v} .
$$

More precisely, letting $P=\sum_{j \in \mathbf{Z}} p_{j}(x, \theta, t) D^{j}$,

$$
\begin{aligned}
\psi(P)_{2 \mu, v} & =\sum_{k=0}^{\infty}\left(\begin{array}{l}
\mu \\
k
\end{array}\right) D^{2 k}\left(p_{v-2 \mu+2 k}\right), \\
\psi(P)_{2 \mu+1, v} & =\sum_{k=0}^{\infty}\left(\begin{array}{l}
\mu \\
k
\end{array}\right)\left\{D^{2 k+1}\left(p_{v-2 \mu+2 k}\right)-(-)^{v} D^{2 k}\left(p_{v-2 \mu-1+2 k}\right)\right\} .
\end{aligned}
$$

From the definition (2.3) and the associativity of the multiplication in $\mathscr{E}$, it is easy to see that $\psi$ is actually an injective algebra homomorphism. (Furthermore $\psi$ becomes a superalgebra homomorphism under an appropriate $\mathbf{Z}_{2}$-gradation of $\operatorname{Mat}(\mathbf{Z} ; \mathscr{P})$.)

Now let us integrate the SKP hierarchy. One first finds a monic super-microdifferential operator (a super-wave operator)

$$
W=\sum_{j=0}^{\infty} w_{j}(x, \theta, t) D^{-j} \in \mathscr{E}_{0}
$$


satisfying

$$
\begin{aligned}
L & =W D W^{-1}, \\
D_{n}(W) & =\varepsilon_{n}\left(B_{n} W-W D^{n}\right), \quad n=1,2, \ldots,
\end{aligned}
$$

where $\varepsilon_{n}=(-)^{n(n+1) / 2}$. Equations (2.4) are referred to as the Sato equations for the SKP hierarchy. Introducing

$$
\Psi=\exp \left(\sum_{n=1}^{\infty} \varepsilon_{n} t_{n} D^{n}\right)
$$

one readily sees that the operator $\tilde{W}=W \cdot \Psi$ solves

$$
D_{n}(\tilde{W})=\varepsilon_{n} B_{n} \tilde{W}, \quad n=1,2, \ldots
$$

Apart from this, consider the following equations:

$$
D_{n}(Y)=\varepsilon_{n} B_{n} Y
$$

where $Y$ is a super-differential operator of the infinite order

$$
Y=\sum_{j=0}^{\infty} y_{j}(x, \theta, t) D^{j} \quad\left(y_{j} \in \mathscr{S}_{\underline{j}}\right),
$$

with an initial condition $\left.Y\right|_{t=0}=1$. Putting $U=\tilde{W}^{-1} Y$, one sees that the coefficients of $U$ are independent of $t$, and that

$$
Y=W Z
$$

Here the operator $Z$ is defined by

$$
Z=\Psi U=\sum_{j \in \mathbf{Z}} z_{j}(x, \theta, t) D^{j}
$$

Taking the (-) part of $(2.5)$ (for $\left.P \in \mathscr{E},(P)_{-}=P-(P)_{+}\right)$yields the following equation:

$$
(W Z)_{-}=0 \text {. }
$$

Introduce a $\mathbf{Z} \times \mathbf{N}^{c}$ matrix $\mathscr{Z}$ by

$$
\mathscr{Z}=\left(\psi(Z)_{\mu v}\right)_{\mu \in \mathbf{Z}, v \in \mathbf{N}^{c}}
$$

Then Eq. (2.6) reads

$$
{ }^{t} \vec{w} \mathscr{Z}=0
$$

where $\vec{w}=\left(w_{-\jmath}\right)_{j \in \mathbf{Z}}, w_{j}=w_{j}(x, \theta, t)$ for $j \geqq 0, w_{j}=0$ for $j<0$. The matrix $\mathscr{Z}$ solves

$$
\begin{aligned}
D_{n}(\mathscr{Z}) & =\Gamma^{n} \mathscr{Z}, \quad n=1,2, \ldots, \\
D(\mathscr{Z}) & =\Lambda \mathscr{Z}-\mathscr{Z}^{\dagger} \Lambda_{\mathbf{N}^{c}},
\end{aligned}
$$

where $\Lambda=\left(\delta_{\mu+1, v}\right)_{\mu, v \in \mathbf{Z}}, \quad \Gamma=\left((-)^{v} \delta_{\mu+1, v}\right)_{\mu, v \in \mathbf{Z}}, \Lambda_{\mathbf{N}^{c}}=\left(\delta_{\mu+1, v}\right)_{\mu, v \in \mathbf{N}^{c}}$ and $\mathscr{Z}^{\dagger}=\left(\psi(Z)_{\mu v}^{\dagger}\right)$ (for $f=f_{0}+f_{1} \in \mathscr{S}=\mathscr{S}_{0} \oplus \mathscr{S}_{1}$, we set $f^{\dagger}=f_{0}-f_{1}$ ). From these equations, the matrix $\mathscr{Z}$ is represented as

$$
\mathscr{Z}=\Phi \cdot \Xi \cdot \exp \left(-\theta \Lambda_{\mathbf{N}^{c}}-x\left(\Lambda_{\mathrm{N}^{c}}\right)^{2}\right)
$$


where

$$
\Phi=\exp \left(\theta \Lambda+x \Lambda^{2}+\sum_{n=1}^{\infty} t_{n} \Gamma^{n}\right),
$$

and $\Xi$ is a constant $\mathbf{Z} \times \mathbf{N}^{c}$ matrix

$$
\Xi=\left(\xi_{\mu v}\right)_{\mu \in \mathbf{Z}, v \in \mathbf{N}^{c}} \in \operatorname{Mat}\left(\mathbf{Z} \times \mathbf{N}^{c} ; \mathscr{A}\right) \text { with } \xi_{\mu v} \in \mathscr{A}_{\underline{\mu+v}} .
$$

One can see that $\xi_{\mu \nu}=\delta_{\mu \nu}$ for $\mu \leqq \nu$. Therefore we have the following proposition.

Proposition 2.1. The coefficients $w_{j}(x, \theta, t) \in \mathscr{S}_{j}(j \geqq 1)$ of a super-wave operator $W \in \mathscr{E}_{0}^{\text {monic }}$ solve a system of an infinite number of linear algebraic equations

$$
{ }^{t} \vec{w} \Phi \Xi=0 .
$$

Equation (2.11) is referred to as the Grassmann equation for the SKP hierarchy. The Grassmann equation has a unique solution for matrix $\Xi$ in the set of superframes:

$$
\begin{aligned}
\operatorname{SFR}\left(\mathbf{N}^{c} ; \mathscr{A}\right)= & \left\{\Xi=\left(\xi_{\mu \nu}\right)_{\mu \in \mathbf{Z}, v \in \mathbf{N}^{c}} \in \operatorname{Mat}\left(\mathbf{Z} \times \mathbf{N}^{c} ; \mathscr{A}\right) \mid \xi_{\mu \nu} \in \mathscr{A}_{\mu+v},\right. \\
& \exists m \in \mathbf{N} \text { such that } \xi_{\mu \nu}=\delta_{\mu \nu} \text { for } \mu<-m, \mu \leqq v, \\
& \left.\xi_{\mu \nu}=0 \text { for }-m \leqq v<0, \mu \leqq-m, \text { and } \varepsilon(\Xi) \text { is of maximal rank }\right\} .
\end{aligned}
$$

The resulting solutions $w_{j}$ belong to the quotient algebra $\mathscr{Q}$ of $\mathscr{S}$. Let $\mathscr{E}_{2}$ be the superalgebra of super-microdifferential operators with coefficients in $\mathscr{Q}$.

Proposition 2.2. For a solution ${ }^{t} \vec{w}$ to the Grassmann equation with $\Xi \in S F R\left(N^{c} ; \mathscr{A}\right)$, set $W=\sum_{j=0}^{\infty} w_{j} D^{-j} \in\left(\mathscr{E}_{2}^{\text {monic }}\right)_{\underline{0}}$. Then the operator $W$ solves the Sato equations (2.3) for the SKP hierarchy with $B_{n}=\left(W D^{n} W^{-1}\right)_{+}$.

We introduce the supergroup $S G L\left(\mathbf{N}^{c} ; \mathscr{A}\right)$ by

$$
\begin{aligned}
S G L\left(\mathbf{N}^{c} ; \mathscr{A}\right)= & \left\{g=\left(g_{\mu \nu}\right)_{\mu, v \in \mathbf{N}^{c}} \in \operatorname{Mat}\left(\mathbf{N}^{c} ; \mathscr{A}\right) \mid g_{\mu \nu} \in \mathscr{A}_{\mu+v},\right. \\
& \exists m \in \mathbf{N} \text { such that } g_{\mu \nu}=\delta_{\mu v} \text { for } \mu \leqq v, \mu<-m, \\
& \left.g_{\mu \nu}=0 \text { for }-m \leqq v<0, \mu \leqq-m, \text { and }\left(\varepsilon\left(g_{\mu v}\right)\right)_{-m \leqq \mu, v<0} \text { is invertible }\right\} .
\end{aligned}
$$

This supergroup acts on the space $\operatorname{SFR}\left(\mathbf{N}^{c} ; \mathscr{A}\right)$ from the right. The universal superGrassmann manifold $U S G M$ is by definition, the quotient space of $S F R\left(\mathbf{N}^{c} ; \mathscr{A}\right)$ :

$$
U S G M=S F R\left(\mathbf{N}^{c} ; \mathscr{A}\right) / S G L\left(\mathbf{N}^{c} ; \mathscr{A}\right) \text {. }
$$

From the formula of solutions to the Grassmann equation (Theorem 2.4), we can see that the biggest cell of USGM,

$$
U S G M^{\phi}=\left\{\boldsymbol{\Xi}=\left(\xi_{\mu \nu}\right) \in S F R\left(\mathbf{N}^{c} ; \mathscr{A}\right) \mid \xi_{\mu \nu}=\delta_{\mu \nu} \text { for } \mu \leqq v\right\} / S G L\left(\mathbf{N}^{c} ; \mathscr{A}\right)
$$

provides super-wave operators with coefficients in $\mathscr{S}$. We denote by $W(\Xi) \in \mathscr{E}_{2}$ the super-wave operator associated with a superframe $\Xi$ in Proposition 2.2. It is obvious that, if two superframes $\Xi, \Xi^{\prime}$ determine the same point in USGM, the associated super-wave operators $W(\Xi)$ and $W\left(\Xi^{\prime}\right)$ coincide. There arises a natural question whether, if two super-wave operators $W(\Xi), W\left(\Xi^{\prime}\right)$ coincide, the super- 
frames $\Xi$ and $\Xi^{\prime}$ determine the same point, namely $\Xi=\Xi^{\prime} \bmod S G L\left(\mathbf{N}^{c} ; \mathscr{A}\right)$. The answer is "yes," at least for superframes that belong to $U S G M^{\phi}$.

Proposition 2.3. Suppose $\Xi, \Xi^{\prime} \in U S G M^{\phi}$, and $W(\Xi)=W\left(\Xi^{\prime}\right)$. Then $\Xi=\Xi^{\prime}$ $\bmod S G L\left(\mathbf{N}^{c} ; \mathscr{A}\right)$.

Proof. We note that, for a superframe $\Xi$ in $U S G M^{\phi}$, there exists a matrix $g \in S G L\left(\mathbf{N}^{c} ; \mathscr{A}\right)$ such that $(\Xi \cdot g)_{\mu \nu}=\widetilde{\xi}_{\mu \nu}\left(\mu \in \mathbf{Z}, v \in \mathbf{N}^{c}\right)$ with $\tilde{\xi}_{\mu \nu}=\delta_{\mu \nu}$ if $\mu \in \mathbf{N}^{c}$. We call such a superframe $\tilde{\Xi}=\left(\tilde{\xi}_{\mu v}\right)_{\mu \in \mathbf{Z}, v \in \mathbf{N}^{c}}$ normalized. Set $W(\Xi)=\sum_{j=0}^{\infty} w_{j}(x, \theta, t) D^{-j}$ $\left(w_{j}(x, \theta, t) \in \mathscr{S}_{j}\right)$. What we have to show is that a normalized superframe $\tilde{\Xi}$ is uniquely determined from the Grassmann equation

$$
{ }^{t} \vec{w} \Phi \widetilde{\Xi}=0
$$

$\left(\vec{w}=\left(w_{-j}\right)_{j \in \mathbf{Z}}, w_{j}=w_{j}(x, \theta, t)\right.$ for $j \geqq 0, w_{j}=0$ for $\left.j<0\right)$. Applying $D^{n}$ to $(2.12)$ and setting $x=\theta=t=0$, we have the following equations:

$$
\overleftrightarrow{w}[n] \widetilde{\Xi}=0 \quad(n=0,1,2, \ldots)
$$

where ${ }^{t} \vec{w}[n]=\left.D^{n}\left({ }^{t} \vec{w} \Phi\right)\right|_{x=\theta=t=0}=\left(w[n]_{j}\right)_{j \in \mathbf{Z}}$ with $w[n]_{j} \in \mathscr{A}_{j+n}, w[n]_{n}=1$ and $w[n]_{j}=0$ for $j>n$. Equations (2.13) imply the orthogonality relations between the vectors $\vec{w}[n]$ and the superframe $\widetilde{\Xi}$. It is easy to see that each column vector of $\widetilde{\Xi}$ is uniquely determined from this orthogonality.

Thus super-wave operators in $\mathscr{E}$ correspond one-to-one to points in $U S G M^{\phi}$.

To study the time evolution of solutions to the SKP hierarchy, introduce an infinite number of supersymmetric derivations;

$$
\begin{aligned}
\bar{D} & =\partial_{\theta}-\theta \partial_{x} \quad\left(\bar{D}^{2}=-\partial_{x}\right), \\
\bar{D}_{2 l} & =\frac{\partial}{\partial t_{2 l}}, \quad \bar{D}_{2 l-1}=\frac{\partial}{\partial t_{2 l-1}}-\sum_{k=1}^{\infty} t_{2 k-1} \frac{\partial}{\partial t_{2 l+2 k-2}} .
\end{aligned}
$$

Consider an even derivation

$$
X=a \frac{\partial}{\partial x}+\zeta \bar{D}+\sum_{n=1}^{\infty} c_{n} \bar{D}_{n}
$$

where $a \in \mathscr{A}_{0}, \zeta \in \mathscr{A}_{1}, c_{n} \in \mathscr{A}_{n} . X$ commutes with the derivations $D$ and $D_{n}$ so that it acts infinitesimally on the solution space of the SKP hierarchy. For a superfield $f \in \mathscr{S}$, one has

$$
\left(e^{X} f\right)(x, \theta, t)=f\left(x^{\prime}, \theta^{\prime}, t^{\prime}\right),
$$

where $\quad x^{\prime}=x+a+\theta \zeta, \quad \theta^{\prime}=\theta+\zeta, \quad t_{2 l-1}^{\prime}=t_{2 l-1}+c_{2 l-1} \quad$ and $\quad t_{2 l}^{\prime}=t_{2 l}+c_{2 l}+$ $\sum_{k=1}^{l} t_{2 k-1} c_{2 l-2 k+1}$. Since the fundamental solution matrix $\Phi(2.10)$ has the multiplicative property with respect to the time evolution, i.e.,

$$
\left(e^{X} \Phi\right)(x, \theta, t)=\Phi(x, \theta, t) \Phi(a, \zeta, c),
$$

the SKP hierarchy is translated to a dynamical system on USGM with the time 
evolution

$$
\Xi \bmod S G L\left(\mathbf{N}^{c} ; \mathscr{A}\right) \rightarrow \Phi(x, \theta, t) \cdot \Xi \bmod S G L\left(\mathbf{N}^{c} ; \mathscr{A}\right) .
$$

In order to solve the Grassmann equation explicitly, we need some algebraic concepts. With a matrix $X=\left(x_{i j}\right)_{i, j \in \mathbf{Z}}$,

$$
\check{X}=\left(X_{\alpha \beta}\right)_{\alpha, \beta=0,1}
$$

is associated, where the blocks are put as $X_{\alpha \beta}=\left(x_{i j}\right)_{l \in 2 \mathbf{Z}+\alpha, j \in 2 \mathbf{Z}+\beta}$. Applying this rearrangement to the Grassmann equation, it is rewritten into the form

$$
\left(\ldots, w_{4}, w_{2}, 1,0, \ldots ; \ldots, w_{3}, w_{1}, 0, \ldots\right) \cdot \check{\Phi} \cdot \check{\Xi}=0 \text {. }
$$

Let $A=\left(A_{\alpha \beta}\right)_{\alpha, \beta=0,1}$ be an invertible matrix with $A_{\alpha \beta} \in \operatorname{Mat}\left(m_{\alpha} \times m_{\beta} ; \mathscr{A}_{\alpha+\beta}\right)$. The invertibility of such a matrix is equivalent to that of the matrices $\varepsilon\left(A_{00}\right)$ and $\varepsilon\left(A_{11}\right)$. A superdeterminant (or the Berezinian) [11] of the matrix $A$ is, by definition,

$$
\text { sdet } A=\operatorname{det}\left(A_{00}-A_{01} A_{11}{ }^{-1} A_{10}\right) / \operatorname{det} A_{11} \text {. }
$$

The inverse of the superdeterminant is given by

$$
\mathrm{s}^{-1} \operatorname{det} A=\operatorname{det}\left(A_{11}-A_{10} A_{00}{ }^{-1} A_{01}\right) / \operatorname{det} A_{00} .
$$

We should remark that a superdeterminant is multiplicative with respect to the product of matrices. By virture of Cramer's formula in linear algebra, one sees that the even unknowns $w_{2 j}$ in (2.14) are expressed in the form of a quotient of superdeterminants. To get the formulas representing the odd unknowns $w_{2 j+1}$, we first look for the formula for $w_{1}$, and consider the first Sato equation $D_{1}(W)=-\left(B_{1} W-W D\right)$. Then we obtain the following theorem.

Theorem 2.4. The coefficients of a super-wave operator attached to a superframe $\Xi \in \operatorname{SFR}\left(\mathbf{N}^{c} ; \mathscr{A}\right)$ are given by

$$
w_{1}=D\left\{\log \left(\operatorname{sdet}\left({ }^{t} \check{\Xi}_{0} \cdot \check{\Phi} \cdot \check{\Xi}\right)\right)\right\}=D_{1}\left\{\log \left(\operatorname{sdet}\left({ }^{t} \check{\Xi}_{0} \cdot \check{\Phi} \cdot \check{\Xi}\right)\right)\right\},
$$

and

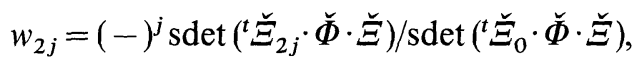

$$
\begin{aligned}
& w_{2 j+1}=(-)^{j}\left\{\left(D+D_{1}\right)\left(\operatorname{sdet}\left({ }^{t} \check{\Xi}_{2 j} \cdot \check{\Phi} \cdot \check{\Xi}\right)\right)\right\} / 2 \operatorname{sdet}\left({ }^{t} \check{\Xi}_{0} \cdot \check{\Phi} \cdot \check{\Xi}\right) \text {, }
\end{aligned}
$$

for $j=0,1,2, \ldots$. Here the frame $\check{\Xi}_{2 j}$ is defined by

$$
\check{\Xi}_{2 j}=\left(\begin{array}{ll}
\Xi_{j} & 0 \\
0 & \Xi_{0}
\end{array}\right),
$$

where $\Xi_{j}=\left(\delta_{\mu v}(\mu \in \mathbf{Z} ; \mu<-j) \mid \delta_{\mu, v+1}(\mu \in \mathbf{Z} ;-j \leqq v<0)\right)$.

Finally we describe the 2-spinor picture of the SKP hierarchy. Let $\tilde{\mathscr{S}}=\mathbf{C}[[x, t]] \otimes \mathscr{A}$ and $\mathscr{E}_{\tilde{\mathscr{S}}}=\tilde{\mathscr{S}}\left(\left(\partial_{x}{ }^{-1}\right)\right)$ be the algebra of microdifferential operators with coefficients in $\tilde{\mathscr{S}}$. Put

$$
\mathscr{L}=\operatorname{Mat}(1 \mid 1 ; \mathbf{C}) \otimes \mathscr{E}_{\tilde{\mathscr{S}}},
$$

whose $\mathbf{Z}_{2}$-gradation $\mathscr{L}=\mathscr{L}_{0} \oplus \mathscr{L}_{1}$ is naturally introduced. We denote by $\tilde{\varepsilon}$ the body map, $\tilde{\varepsilon}: \tilde{\mathscr{S}} \rightarrow \mathscr{R}$, which is defined in the same way as before. The same notation 
$\tilde{\varepsilon}$ expresses the body map $\mathscr{E}_{\tilde{\mathscr{T}}} \rightarrow \mathscr{E}_{\mathscr{P}}$, which further extends to the body map $\tilde{\varepsilon}_{\mathscr{E}}: \mathscr{L} \rightarrow \mathscr{E}_{\mathscr{R}} \oplus \mathscr{E}_{\mathscr{R}}$. The 2-spinor representation is a superalgebra homomorphism $\pi: \mathscr{E} \rightarrow \mathscr{L}$ defined by

$$
\begin{aligned}
& \pi(\theta)=\left(\begin{array}{ll}
0 & 0 \\
1 & 0
\end{array}\right), \quad \pi(D)=\left(\begin{array}{cc}
0 & 1 \\
\partial_{x} & 0
\end{array}\right), \\
& \pi(f)=\operatorname{diag}\left(f,(-)^{a} f\right) \text { for } f \in \tilde{\mathscr{S}}_{a} .
\end{aligned}
$$

We consider the Sato equation (2.4) with $n=2 l$ in the 2-spinor representation. Let $W$ be a super-wave operator in $\mathscr{E}$ and $\pi(W)=\left(W_{i j}\right)_{i, j=0,1}$. Each entry $W_{i j}$ belongs to $\left(\mathscr{E}_{\tilde{\mathscr{F}}}\right)_{i+j}$, and the body part of the diagonal entries satisfy

$$
\frac{\partial}{\partial t_{2 l}} \tilde{\varepsilon}\left(W_{i i}\right)=(-)^{l}\left\{\tilde{\varepsilon}\left(B_{2 l, i i}\right) \tilde{\varepsilon}\left(W_{i i}\right)-\tilde{\varepsilon}\left(W_{i i}\right) \partial_{x}{ }^{l}\right\},
$$

where $\pi\left(B_{2 l}\right)=\left(B_{2 l, i j}\right)_{i, j=0,1}$. These are nothing but the Sato equations for the KP hierarchy. Therefore we have the following proposition.

Proposition 2.5. Let $\mathscr{W}_{\mathrm{SKP}}^{\phi}$ be the space of super-wave operators in $\mathscr{E}$ of the SKP hierarchy, and $\mathscr{W}_{\mathrm{KP}}^{\phi}$ be the space of wave operators in $\mathscr{E}_{\mathscr{R}}$ of the $\mathrm{KP}$ hierarchy. Then we have the projection $\rho=\tilde{\varepsilon}_{\mathscr{L}}{ }^{\circ} \pi$,

$$
\rho: \mathscr{W}_{\mathrm{SKP}}^{\phi} \rightarrow \mathscr{W}_{\mathrm{KP}}^{\phi} \times \mathscr{W}_{\mathrm{KP}}^{\phi} .
$$

\section{Super-Laplace Transform and Bilinear Residue Formula}

First we discuss the concept of the "formal adjoint" in $\mathscr{E}_{\mathrm{C}[[x, \theta]] \otimes \mathscr{A}}$. The superintegration of a superfield $f(x, \theta)=u(x)+\theta v(x)$ is, by definition,

$$
\int \Delta(d x / d \theta) f(x, \theta)=\int d x v(x)
$$

where $\Delta(d x / d \theta)$ is the (1|1)-dimensional volume form (an odd quantity). For a given $P=P(x, \theta, D) \in \mathscr{E}_{\mathrm{C}[[x, \theta]] \otimes \mathscr{A}}$, the formal adjoint operator $P^{*}=P^{*}(x, \theta, D) \in \mathscr{E}_{\mathrm{C}[[x, \theta]] \otimes \mathscr{A}}$ is introduced through

$$
\begin{aligned}
& \int \Delta(d x / d \theta) P(x, \theta, D)(f(x, \theta)) \cdot g(x, \theta) \\
& \quad=\int \Delta(d x / d \theta) f(x, \theta) \cdot P^{*}(x, \theta, D)(g(x, \theta)),
\end{aligned}
$$

for $f(x, \theta), g(x, \theta) \in(\mathbb{C}[[x, \theta]] \otimes \mathscr{A})_{\underline{0}}$. Then we have

$$
\left(w D^{n}\right)^{*}=(-)^{a n} \varepsilon_{n} D^{n} \cdot w \quad\left(w \in(\mathbb{C}[[x, \theta]] \otimes \mathscr{A})_{a}, n \in \mathbf{Z}\right),
$$

and, in general,

$$
\left(P_{1} P_{2}\right)^{*}=(-)^{a_{1} a_{2}} P_{2}{ }^{*} P_{1} * \quad\left(P_{j} \in\left(\mathscr{E}_{\mathbb{C}[[x, \theta]] \otimes \infty \mathcal{A}}\right)_{a_{j}}\right) .
$$

We introduce a super-wave function and its dual version. Let

$$
H(x, \theta, t, \lambda, \xi)=x \lambda+\sum_{l=1}^{\infty}(-)^{l} t_{2 l} \lambda^{l}+(\xi+h(t, \xi))\left(\theta+\lambda^{-1} h(t, \xi)\right),
$$

where

$$
h(t, \lambda)=\sum_{l=1}^{\infty}(-)^{l} t_{2 l-1} \lambda^{l}
$$


Here $(\lambda, \xi)$ are regarded as (1|1)-dimensional spectral parameters. For a super-wave operator $W=W(x, \theta, t, D)=\sum_{j=0}^{\infty} w_{j}(x, \theta, t) D^{-j}\left(w_{0}=1\right)$, define a super-wave function and its dual by

$$
\begin{gathered}
w(x, \theta, t, \lambda, \xi)=W(x, \theta, t, D)(\exp (H(x, \theta, t, \lambda, \xi))), \\
w^{*}(x, \theta, t, \lambda, \xi)=W^{*}(x, \theta, t, D)^{-1}(\exp (-H(x, \theta, t, \lambda, \xi))) .
\end{gathered}
$$

Note that

$$
\begin{aligned}
D^{2}(\exp H) & =\lambda \exp H, \quad D_{n}(\exp H)=\varepsilon_{n} D^{n}(\exp H), \\
D^{-2 \mu}(\exp H) & =\lambda^{-\mu} \exp H, \quad D^{-2 \mu+1}(\exp H)=\lambda^{-\mu}(\lambda \theta-\xi-h) \exp H .
\end{aligned}
$$

By the Sato equations (2.4), a super-wave function and its dual satisfy the linear equations

$$
D_{n}(w)=\varepsilon_{n} B_{n}(w), \quad D_{n}\left(w^{*}\right)=-\varepsilon_{n} B_{n}^{*}\left(w^{*}\right) .
$$

We consider the duality of the super-Laplace transform. Let $\mathbf{V}=\mathbf{V}_{\mathbf{C}} \otimes \mathscr{A}$ be an $\mathscr{A}$-module, where

$$
\mathbf{V}_{\mathbf{C}}=\left\{\sum_{-\infty \ll \mu<\infty} e_{\mu} c_{\mu} \mid c_{\mu} \in \mathbf{C}\right\}=\left(\mathbf{V}_{\mathbf{C}}\right)_{\underline{0}} \oplus\left(\mathbf{V}_{\mathbf{C}}\right)_{\underline{1}}
$$

with basis elements $e_{\mu} \in\left(\mathbf{V}_{\mathbf{C}}\right)_{\mu}$. The $\mathscr{A}$-module $\mathbf{V}$ has a natural pairing $\langle\rangle:, \mathbf{V} \otimes \mathbf{V} \rightarrow \mathscr{A}$ defined by

$$
\left\langle e_{\mu}, e_{-v-1}\right\rangle=\delta_{\mu v}, \quad\langle u a, v\rangle=\langle u, a v\rangle \text { and }\langle u, v a\rangle=\langle u, v\rangle a,
$$

for $u, v \in \mathbf{V}, a \in \mathscr{A}$. We identify an element $u=\sum_{-\infty \ll \mu<\infty} e_{\mu} a_{\mu}$ with a super-microfunction $u(x, \theta)=\sum_{-\infty \ll \mu<\infty} \delta^{(\mu)}(x, \theta) a_{-\mu-1}$, where we have defined the super-delta function by $\delta(x, \theta)=\bar{\theta} \delta(x)$, and $\delta^{(\mu)}(x, \theta)=D^{\mu}(\delta(x, \theta))(\mu \in \mathbf{N})$. More precisely, one has

$$
\begin{aligned}
\delta^{(2 \mu)}(x, \theta) & =\theta \partial_{x}{ }^{\mu}(\delta(x)), \quad \delta^{(2 \mu+1)}(x, \theta)=\partial_{x}{ }^{\mu}(\delta(x)), \\
\delta^{(-2 \mu-2)}(x, \theta) & =\theta x^{\mu} Y(x) / \mu !, \quad \delta^{(-2 \mu-1)}(x, \theta)=x^{\mu} Y(x) / \mu !,
\end{aligned}
$$

for $\mu \in \mathbf{N}$, where $Y(x)$ is the Heaviside function. Set

$$
\begin{aligned}
\mathbf{V}^{\phi} & =\left\{u=\sum_{-\infty \ll \mu<0} e_{\mu} a_{\mu}\right\} \\
& =\left\{u(x, \theta)=\sum_{0 \leqq \mu \ll \infty} \delta^{(\mu)}(x, \theta) a_{-\mu-1}\right\} .
\end{aligned}
$$

Define the super-Laplace transform of $\delta^{(\mu)}(x, \theta)$ by

$$
\int \Delta(d x / d \theta) \exp (-\lambda x-\xi \theta) \delta^{(\mu)}(x, \theta)=\left(\xi+\lambda \frac{\partial}{\partial \xi}\right)^{\mu}(1) \quad(\mu \in \mathbf{Z}) .
$$

Note that $(\xi+\lambda(\partial / \partial \xi))^{2}=\lambda$. Hence we can rewrite (3.4) as

$$
\hat{e}_{\mu}(\lambda, \xi)=\int \Delta(d x / d \theta) \exp (-\lambda x-\xi \theta) e_{\mu}= \begin{cases}\xi \lambda^{-\mu / 2-1} & (\mu: \text { even }) \\ \lambda^{-(\mu+1) / 2} & (\mu \text { :odd })\end{cases}
$$


For a general element $u=\sum e_{\mu} a_{\mu}$, we set $\hat{u}(\lambda, \xi)=\sum \hat{e}_{\mu}(\lambda, \xi) a_{\mu}$. By the super-Laplace transform we get the identification

$$
\mathbf{V} \ni u(x, \theta) \stackrel{\sim}{\longrightarrow} \hat{u}(\lambda, \xi) \in \mathbf{C}\left(\left(\lambda^{-1}, \xi\right)\right) \otimes \mathscr{A} \text {. }
$$

For $\hat{u}(\lambda, \xi)=\sum \lambda^{\mu} a_{\mu}+\xi \sum \lambda^{\mu} b_{\mu} \in \mathbf{C}\left(\left(\lambda^{-1}, \xi\right)\right) \otimes \mathscr{A}$, set

$$
\operatorname{Res}_{\lambda=\infty}(\Delta(d \lambda / d \xi) \hat{u}(\lambda, \xi))=b_{-1} .
$$

To show the bilinear residue formula, we have to present some lemmas on the residue calculus, the super-Laplace inverse transform and the formal adjoint of operators.

Lemma 3.1. For $u, v \in \mathbf{V}$, we have

$$
\langle u, v\rangle=\operatorname{Res}_{\lambda=\infty}(\Delta(d \lambda / d \xi) \hat{u}(\lambda, \xi) \hat{v}(\lambda, \xi)) .
$$

Proof. It is easy to see that

$$
\operatorname{Res}_{\lambda=\infty}\left(\Delta(d \lambda / d \xi) \hat{e}_{\mu}(\lambda, \xi) \hat{e}_{-v-1}(\lambda, \xi)\right)=0
$$

if $\mu-v$ is odd. If $\mu-v$ is even, then

$$
\operatorname{Res}_{\lambda=\infty}\left(\Delta(d \lambda / d \xi) \hat{e}_{\mu}(\lambda, \xi) \hat{e}_{-v-1}(\lambda, \xi)\right)=\operatorname{Res}_{\lambda=\infty}\left(\Delta(d \lambda / d \xi) \xi \lambda^{-1+(\mu-v) / 2}\right)=\delta_{\mu v}
$$

For a super-microdifferential operator $P \in \mathscr{E}_{\mathrm{C}[[x, \theta]] \otimes \mathscr{A}}$, we define a superdifferential operator of infinite order $\hat{P} \in \mathscr{D}_{\mathbf{C}\left(\left(\hat{i}^{-1}, \xi\right)\right) \otimes \mathscr{A}}^{\infty}$ through the super-Laplace transform: $\hat{P}(\hat{u}(\lambda, \xi))=(P u)^{\hat{n}}(\lambda, \xi)$. For example, $\left(D^{\mu}\right)^{\hat{\prime}}=(\hat{D})^{\mu}=(\xi+\lambda(\partial / \partial \xi))^{\mu}$ for $\mu \in \mathbf{Z}$, and $(\partial / \partial \theta) \hat{=}$. And $\hat{f}=f\left(-d / d \lambda,(-)^{a-1} d / d \xi\right)$ for a superfield $f=f(x, \theta) \in$ $(\mathbf{C}[[x, \theta]] \otimes \mathscr{A})_{a}$.

For a column vector $\left(a_{\mu}\right)_{\mu \in \mathbf{Z}}$ that corresponds to an element $u=\sum e_{\mu} a_{\mu}$ of $\mathbf{V}$, set $\left(a_{\mu}(x, \theta)\right)_{\mu \in \mathbf{Z}}=\exp \left(\theta \Lambda+x \Lambda^{2}\right)\left(a_{\mu}\right)_{\mu \in \mathbf{Z}}$. Then one sees that

$$
\begin{aligned}
a_{\mu}(x, \theta) & =\operatorname{Res}_{\lambda=\infty}\left(\Delta(d \lambda / d \xi) \exp (\lambda x+\xi \theta) \hat{D}^{\mu}(\hat{u}(\lambda, \xi))\right), \\
D\left(a_{\mu}(x, \theta)\right) & =a_{\mu+1}(x, \theta) .
\end{aligned}
$$

Lemma 3.2. An element $u \in \mathbf{V}$ belongs to $\mathbf{V}^{\phi}$ if and only if

$$
\operatorname{Res}_{\lambda=\infty}(\Delta(d \lambda / d \xi) \exp (\lambda x+\xi \theta) \hat{u}(\lambda, \xi))=0 .
$$

Proof. A direct consequence of (3.6) and (3.7).

Lemma 3.3. Let $p(x, \theta) \in(\mathbf{C}[[x, \theta]] \otimes \mathscr{A})_{\underline{v}}$. If $\mu-v$ is even, then

$$
\left(\hat{p} \hat{D}^{\mu}\right)^{*}=\left(\hat{D}^{\mu}\right)^{*} p\left(\frac{\partial}{\partial \lambda}, \frac{\partial}{\partial \xi}\right) .
$$

Proof. Let both $\mu$ and $v$ be odd. For even superfields $f(\lambda, \xi)$ and $g(\lambda, \xi)$,

$$
\begin{aligned}
\int \Delta(d \lambda / d \xi)\left(\left(\hat{p} \hat{D}^{\mu}\right)(f)\right) g & =\int \Delta(d \lambda / d \xi)\left(\hat{D}^{\mu}(f)\right) p\left(\frac{\partial}{\partial \lambda}, \frac{\partial}{\partial \xi}\right)(g) \\
& =\int \Delta(d \lambda / d \xi) f\left(\hat{D}^{\mu}\right)^{*} p\left(\frac{\partial}{\partial \lambda}, \frac{\partial}{\partial \xi}\right)(g) .
\end{aligned}
$$

The other case ( $\mu, v$ are even) is similarly checked. 
Lemma 3.4. $\left(\hat{D}^{\mu}\right)^{*}(\exp (\lambda x+\xi \theta))=(-D)^{\mu}(\exp (\lambda x+\xi \theta))$,

$$
\left(\hat{D}^{\mu}\right)(\exp (-\lambda x-\xi \theta))=(-)^{\mu}\left(D^{\mu}\right)^{*}(\exp (-\lambda x-\xi \theta)) .
$$

Proof is straightforward.

Lemma 3.5. Let $P \in \mathscr{E}_{\mathrm{C}[[x, \theta]] \otimes \mathscr{A}}$ is an even operator. Then

$$
\begin{aligned}
P(\exp (\lambda x+\xi \theta)) & =\hat{P}^{*}(\exp (\lambda x+\xi \theta)), \\
P^{*}(\exp (-\lambda x-x \theta)) & =\hat{P}(\exp (-\lambda x-\xi \theta)) .
\end{aligned}
$$

Proof. Without loss of generality, we can set $P=p(x, \theta) D^{\mu}$, where $p(x, \theta)$ has the parity $\mu$.

$$
\begin{aligned}
\left(\hat{p} \hat{D}^{\mu}\right)^{*}(\exp (\lambda x+\xi \theta)) & =\left(\hat{D}^{\mu}\right)^{*} p\left(\frac{\partial}{\partial \lambda}, \frac{\partial}{\partial \xi}\right)(\exp (\lambda x+\xi \theta)) \\
& =\left(\hat{D}^{\mu}\right)^{*}(p(x, \theta)(\exp (\lambda x+\xi \theta))) \\
& =p(x, \theta)(-)^{\mu}\left(\hat{D}^{\mu}\right)^{*}(\exp (\lambda x+\xi \theta)) \\
& =p(x, \theta) D^{\mu}(\exp (\lambda x+\xi \theta)) .
\end{aligned}
$$

The other one is similarly checked.

Now we can state the bilinear residue formula (BRF) in $\mathscr{E}_{\mathrm{C}[[x, \theta]] \otimes \mathscr{A}}$.

Theorem 3.6. Let $P, Q \in \mathscr{E}_{\mathrm{C}[[x, \theta]] \otimes \mathscr{A}}$ are even operators. Then $P Q \in \mathscr{D}_{\mathrm{C}[[x, \theta]] \otimes \mathscr{Q}}$ if and only if the BRF

$$
\operatorname{Res}_{\lambda=\infty}\left(\Delta(d \lambda / d \xi) P(\exp (\lambda x+\xi \theta)) Q^{*}\left(\exp \left(-\lambda x^{\prime}-\xi \theta^{\prime}\right)\right)=0\right.
$$

holds for any $(x, \theta),\left(x^{\prime}, \theta^{\prime}\right)$.

Proof. The condition $P Q \in \mathscr{D}$ is equivalent to $P Q\left(e_{-k-1}\right) \in \mathbf{V}^{\phi}$ for all $k \in \mathbf{N}$, and also to

$$
\operatorname{Res}_{\lambda=\infty}\left(\Delta(d \lambda / d \xi)((P Q) \hat{)}) *\left(\exp (\lambda x+\xi \theta) \hat{e}_{-k-1}(\lambda, \xi)\right)=0\right.
$$

for all $k \in \mathbf{N}$. Here we recall that $\hat{e}_{-k-1}(\lambda, \xi)=\lambda^{k / 2}$ ( $k$ :even), $=\xi \lambda^{(k-1) / 2}(k:$ odd $)$. Multiplying $(1 / k !)\left(-x^{\prime}\right)^{k / 2}(k$ :even $),\left(\left(-\theta^{\prime}\right) /(k-1) !\right)\left(-x^{\prime}\right)^{(k-1) / 2}(k$ :odd) from the right of (3.8), and summing up over $k \in \mathbf{N}$, we have a generating function expression

$$
\operatorname{Res}_{\lambda=\infty}\left(\Delta(d \lambda / d \xi)\left((P Q)^{\hat{\prime}}\right)^{*}(\exp (\lambda x+\xi \theta)) \exp \left(-\lambda x^{\prime}-\xi \theta^{\prime}\right)\right)=0 .
$$

This and Lemma 3.5 complete the proof.

We are in the position to state one of our main results in this paper.

Theorem 3.7. Formal superfields $w(x, \theta, t, \lambda, \xi)$ and $w^{*}(x, \theta, t, \lambda, \xi)$ of the form (3.1) and (3.2) are a super-wave function and its dual for the SKP hierarchy if and only if they satisfy the $\mathrm{BRF}$

$$
\operatorname{Res}_{\lambda=\infty}\left(\Delta(d \lambda / d \xi) w\left(x^{\prime}, \theta^{\prime}, t^{\prime}, \lambda, \xi\right) w^{*}(x, \theta, t, \lambda, \xi)\right)=0
$$

for any $(x, \theta, t)$ and $\left(x^{\prime}, \theta^{\prime}, t^{\prime}\right)$.

Proof. From Theorem 3.6 it follows that

$$
\operatorname{Res}_{\lambda=\infty}\left(\Delta(d \lambda / d \xi)\left(D^{\prime}\right)^{\mu}\left(w\left(x^{\prime}, \theta^{\prime}, t, \lambda, \xi\right)\right) w^{*}(x, \theta, t, \lambda, \xi)\right)=0 .
$$


Equations (3.3) show that, for any multi-index $\alpha=\left(\alpha_{1}, \alpha_{2}, \ldots\right)$,

$$
\operatorname{Res}_{\lambda=\infty}\left(\Delta(d \lambda / d \xi)\left(D_{t}\right)^{\alpha}\left(w\left(x^{\prime}, \theta^{\prime}, t, \lambda, \xi\right)\right) w^{*}(x, \theta, t, \lambda, \xi)\right)=0,
$$

where we have put $\left(D_{t}\right)^{\alpha}=D_{t_{1}}{ }^{\alpha_{1}} D_{t_{2}}{ }^{\alpha_{2}} \cdots$. The BRF (3.9) follows as a generating function expression of (3.10). Conversely, if (3.9) is satisfied, we have

$$
\operatorname{Res}_{\lambda=\infty}\left(\Delta(d \lambda / d \xi)\left(D_{n}-\varepsilon_{n} B_{n}\right)(w(x, \theta, t, \lambda, \xi)) w^{*}\left(x^{\prime}, \theta^{\prime}, t, \lambda, \xi\right)\right)=0 .
$$

Note that

$$
\left(D_{n}-\varepsilon_{n} B_{n}\right)(w(x, \theta, t, \lambda, \xi))=\left(D_{n}(W) W^{-1}-\varepsilon_{n} B_{n}^{c}\right) W(\exp (H(x, \theta, t, \lambda, \xi))),
$$

where $B_{n}^{c}=-\left(W D^{n} W^{-1}\right)_{-}$. Then Theorem 3.6 implies that $D_{n}(W) W^{-1}-\varepsilon_{n} B_{n}^{c} \in \mathscr{D}$, however, which should be of negative order, by definition. Thus we get $D_{n}(W)=$ $\varepsilon_{n} B_{n}^{c} W$, that are equivalent to the Sato equations.

\section{Ortho-Symplectic SKP Hierarchy}

In this section we discuss the OSp-SKP hierarchy. Let $W$ be a super-wave operator in $\mathscr{E}$ for the SKP hierarchy. The OSp-SKP hierarchy is defined by the condition:

$$
D^{-1} W^{*} D=W^{-1}
$$

in the OSp-sector $t_{4 n+1}=t_{4 n+4}=0$ for $n=0,1,2, \ldots$ The Sato equations read

$$
\begin{aligned}
& D_{4 n+2}(W)=-\left(B_{4 n+2} W-W D^{4 n+2}\right), \\
& D_{4 n+3}(W)=B_{4 n+3} W-W D^{4 n+3}, \quad n=0,1,2, \ldots
\end{aligned}
$$

with the symmetries

$$
D^{-1}\left(B_{4 n+2}\right) * D=-B_{4 n+2}, \quad D^{-1}\left(B_{4 n+3}\right) * D=B_{4 n+3},
$$

in the OSp-sector. In this section the time variables $t$ are supposed to be restricted in the OSp-sector.

We define a super-wave function for a solution $W=\sum_{j=0}^{\infty} w_{j}(x, \theta, t) D^{-j}$ to the OSp-SKP hierarchy by

$$
w(x, \theta, t, \lambda, \xi)=W(\exp \tilde{H})
$$

where

$$
\begin{aligned}
\tilde{H}= & \tilde{H}(x, \theta, t, \lambda, \xi)=x \lambda-\sum_{n=0}^{\infty} t_{4 n+2} \lambda^{2 n+1} \\
& +\left(\xi+\sum_{n=0}^{\infty} t_{4 n+3} \lambda^{2 n+2}\right)\left(\theta+\sum_{n=0}^{\infty} t_{4 n+3} \lambda^{2 n+1}\right) .
\end{aligned}
$$

We also put

$$
v(x, \theta, t, \lambda, \xi)=W D^{-1}(\exp (-\tilde{H})) .
$$

Theorem 4.1. The superfields of the form (4.4) and (4.5) are super-wave functions of 
the OSp-SKP hierarchy if and only if they enjoy

$$
\operatorname{Res}_{\lambda=\infty}\left(\Delta(d \lambda / d \xi) w(x, \theta, t, \lambda, \xi) v\left(x^{\prime}, \theta^{\prime}, t^{\prime}, \lambda, \xi\right)\right)=1
$$

for any $(x, \theta, t)$ and $\left(x^{\prime}, \theta^{\prime}, t^{\prime}\right)$.

Proof. Suppose that $W$ is a solution to the OSp-SKP hierarchy. From the BRF for the SKP hierarchy, we get

$$
\begin{aligned}
0 & =\operatorname{Res}_{\lambda=\infty}\left(\Delta(d \lambda / d \xi) W\left(e^{\tilde{H}(x, \theta, t, \lambda, \xi)}\right)\left(W^{*}\right)^{-1}\left(e^{-\tilde{H}\left(x^{\prime}, \theta^{\prime}, t^{\prime}, \lambda, \xi\right)}\right)\right) \\
& =\operatorname{Res}_{\lambda=\infty}\left(\Delta(d \lambda / d \xi) W\left(e^{\tilde{H}(x, \theta, t, \lambda, \xi)}\right) D^{\prime} W D^{\prime-1}\left(e^{-\tilde{H}\left(x^{\prime}, \theta^{\prime}, t^{\prime}, \lambda, \xi\right)}\right)\right) \\
& =\operatorname{Res}_{\lambda=\infty}\left(\Delta(d \lambda / d \xi) W\left(e^{\tilde{H}(x, \theta, t, \lambda, \xi)}\right) D^{\prime}\left(v\left(x^{\prime}, \theta^{\prime}, t^{\prime}, \lambda, \xi\right)\right) .\right.
\end{aligned}
$$

The superfield $v\left(x^{\prime}, \theta^{\prime}, t^{\prime}, \lambda, \xi\right)$ solves the linear differential equation $D_{n}{ }^{\prime}(v)=\varepsilon_{n} B_{n}{ }^{\prime}(v)$ so that one has

$$
D_{n}{ }^{\prime}\left(\operatorname{Res}_{\lambda=\infty}\left(\Delta(d \lambda / d \xi) w(x, \theta, t, \lambda, \xi) v\left(x^{\prime}, \theta^{\prime}, t^{\prime}, \lambda, \xi\right)\right)=0 .\right.
$$

Namely, the left-hand side of (4.6) is independent of $t^{\prime}$. Putting $x=x^{\prime}, \theta=\theta^{\prime}$ and $t=t^{\prime}$ therein one gets the equality (4.6). Conversely suppose that (4.6) holds. Then the second equation in (4.7), we get $W \cdot\left(D W D^{-1}\right)^{*}=1$ by means of Theorem 3.6. This completes the proof.

Now we discuss the 2-spinor representation of the OSp-SKP hierarchy. We introduce the super-adjoint in $\mathscr{L}$ by, for $P=\left(P_{i j}\right)_{i, j=0,1}$,

$$
P^{\#}=\left(\begin{array}{rr}
P_{00}^{*} & (-)^{a} P_{10}^{*} \\
(-)^{a+1} P_{01}^{*} & P_{11}^{*}
\end{array}\right) \quad\left(P \in \mathscr{L}_{\underline{a}}\right),
$$

where $P_{i j}^{*}$ is the formal adjoint operator of $P_{i j}$ in $\mathscr{E}_{\tilde{\mathscr{S}}}$. We define Lie superalgebra $\operatorname{Dsp}\left(\mathscr{E}_{\tilde{\mathscr{S}}}\right)$ by

$$
\begin{aligned}
\mathfrak{D} \mathfrak{s p}\left(\mathscr{E}_{\tilde{\mathcal{S}}}\right) & =\mathfrak{v} \mathfrak{s p}\left(\mathscr{E}_{\tilde{\mathscr{S}}}\right)_{\underline{\underline{0}}} \oplus \mathfrak{v} \mathfrak{p p}\left(\mathscr{E}_{\tilde{\mathcal{S}}}\right)_{\underline{1}}, \\
\mathfrak{D} \mathfrak{s p}\left(\mathscr{E}_{\tilde{\mathscr{S}}}\right)_{\underline{a}} & =\left\{P \in \mathscr{L}_{\underline{a}} \mid M^{-1} P^{\#} M=(-)^{a} P\right\},
\end{aligned}
$$

where $M=\operatorname{diag}\left(\partial_{x}, 1\right)$. The corresponding Lie supergroup $\operatorname{OSp}\left(\mathscr{E}_{\tilde{\mathscr{S}}}\right)$ is introduced by

$$
\operatorname{OSp}\left(\mathscr{E}_{\tilde{S}}\right)=\left\{P \in \mathscr{L}_{\underline{0}} \mid P \text { is invertible and } M^{-1} P^{\#} M=P^{-1}\right\} .
$$

Proposition 4.2. Let $\mathscr{W}_{\mathrm{OSp}}^{\phi}$ be the space of super-wave operators in $\mathscr{E}$ of the OSp-SKP hierarchy, and $\mathscr{W}_{\mathrm{OSp}}^{\phi}$ (respectively $\mathscr{W}_{\mathrm{CKP}}^{\phi}$ ) be the space of wave operators in $\mathscr{E}_{\mathscr{R}}$ of the BKP (respectively CKP) hierarchy. Then we have the projection

$$
\left.\rho\right|_{\mathscr{W}_{\mathrm{OSp}}^{\phi}}: \mathscr{W}_{\mathrm{OSp}}^{\phi} \rightarrow \mathscr{W}_{\mathrm{BKP}}^{\phi} \times \mathscr{W}_{\mathrm{CKP}}^{\phi},
$$

where the map $\rho$ was introduced in Proposition 2.5.

Proof. First we note that

$$
\left(\begin{array}{ll}
0 & 1 \\
1 & 0
\end{array}\right) \pi(X)^{\#}\left(\begin{array}{ll}
0 & 1 \\
1 & 0
\end{array}\right)=(-)^{a} \pi\left(X^{*}\right) \quad\left(X \in \mathscr{E}_{\underline{a}}\right)
$$

and

$$
\left(\begin{array}{ll}
0 & 1 \\
1 & 0
\end{array}\right) \pi(D)=M
$$


Let $W \in \mathscr{W}_{\text {osp }}^{\phi}$. Then the condition (4.1) reads in the 2-spinor representation

$$
M^{-1} \pi(W)^{\#} M=\pi(W)^{-1},
$$

namely, $\pi(W) \in \operatorname{OSp}\left(\mathscr{E}_{\tilde{\mathcal{S}}}\right)$. Let $\pi(W)=\left(W_{i j}\right)_{i, j=0,1}$. Applying the body map $\tilde{\varepsilon}_{\mathscr{E}}$ to the both sides of $(4.8)$, we see that $\tilde{\varepsilon}\left(W_{00}\right)$ (respectively $\left.\tilde{\varepsilon}\left(W_{11}\right)\right)$ satisfies the BKP (respectively CKP) condition.

In the rest of this section we characterize solutions to the OSp-SKP hierarchy in terms of superframes in $U S G M^{\phi}$.

Proposition 4.3. Let $P$ be an operator in $\mathscr{E}_{a}$. Then it follows that

$$
\psi^{\vee}\left(P^{*}\right)=(-)^{a} \text { offdiag }\left({ }^{t} K,{ }^{t} K\right)^{s t} \psi^{\vee}(P) \text { offdiag }(K, K),
$$

where offdiag $(A, B)$ stands for $\left(\begin{array}{cc}0 & A \\ B & 0\end{array}\right)$ with $A, B \in M a t(\mathbf{Z} \times \mathbf{Z})$, and $K=\Lambda J$, $J=\left((-)^{\mu} \delta_{\mu,-v}\right)_{\mu, v \in \mathbf{Z}}$. The symbol $\psi^{\vee}(P)$ means the "check" of the matrix $\psi(P)$ (cf. Sect. 2), and "st" is the supertransposition of a matrix (cf. [7]).

Proof. We only have to show the claim in the case of $P=u D^{j} u \in \mathscr{S}_{a}$. For $u=f(x, t)+\theta g(x, t)$,

$$
\psi^{\vee}(u)=\left(\begin{array}{lc}
\sigma(f)+\theta \sigma(g) & 0 \\
\theta \sigma\left(f_{x}\right)+\sigma(g) & (-)^{a}(\sigma(f)+\theta \sigma(g))
\end{array}\right),
$$

where $\sigma(f)=\left(\left(\begin{array}{c}i \\ i-k\end{array}\right) f^{(i-k)}(x, t)\right)_{i, k \in \mathbf{Z}},\left(\begin{array}{c}m \\ n\end{array}\right)=0$ for $n<0$. By a simple calculation we have

$$
\text { offdiag }\left({ }^{t} K,{ }^{t} K\right)^{s t} \psi^{\vee}(u) \operatorname{offdiag}(K, K)=(-)^{a} \psi^{\vee}(u) \text {. }
$$

Since $\psi^{\vee}(D)=\left(\begin{array}{ll}0 & 1 \\ \Lambda & 0\end{array}\right)$, it follows that

$$
\psi^{\vee}\left(\left(D^{j}\right)^{*}\right)=(-)^{j} \text { offdiag }\left({ }^{t} K,{ }^{t} K\right)^{s t} \psi^{\vee}\left(D^{j}\right) \operatorname{offdiag}(K, K) .
$$

Then we have

$$
\psi^{\vee}\left(\left(u D^{j}\right)^{*}\right)=(-)^{a+j} \text { offdiag }\left({ }^{t} K,{ }^{t} K\right)^{s t} \psi^{\vee}\left(u D^{j}\right) \operatorname{offdiag}(K, K) .
$$

Now we introduce the Lie supergroup $O S p(\mathscr{S})$ [9],

$$
\begin{aligned}
\operatorname{OSp}(\mathscr{S})= & \left\{A=\left(A_{\alpha \beta}\right)_{\alpha, \beta=0,1} \mid A_{\alpha \beta} \in \operatorname{Mat}\left(\mathbf{Z} \times \mathbf{Z}, \mathscr{S}_{\alpha+\beta}\right) \text { and } \varepsilon(A)\right. \text { is } \\
& \text { invertible and } \left.\operatorname{diag}\left(J,-{ }^{t} K\right)^{s t} \check{A} \operatorname{diag}(J,-K)=\check{A}^{-1} \cdot\right\} .
\end{aligned}
$$

Notice that for an operator $U$ in $\mathscr{E}_{0}$, the condition

$$
D^{-1} U^{*} D=U^{-1},
$$

is equivalent to that $\psi^{\vee}(U) \in O S p(\mathscr{S})$. We introduce the following inner products $\langle,\rangle_{B}\langle,\rangle_{C}$,

$$
\langle\overrightarrow{f,}, \vec{g}\rangle_{B}=\sum_{j \in \mathbf{Z}}(-)^{j} f_{j} g_{-j},\langle\overrightarrow{f,} \vec{g}\rangle_{C}=\sum_{j \in \mathbf{Z}}(-)^{j+1} f_{j} g_{-j-1},
$$


for column vectors $\vec{f}=\left(f_{j}\right)_{j \in \mathbf{Z}}, \vec{g}=\left(g_{j}\right)_{j \in \mathbf{Z}}$. Put $\psi^{\vee}(U)=\left(\left(u_{i j}^{\alpha \beta}\right)_{i, j \in \mathbf{Z}}\right)_{\alpha, \beta=0,1}$ and $\vec{u}_{j}^{\alpha \beta}=\left(u_{i, j}^{\alpha \beta}\right)_{i \in \mathbf{Z}}$. If $\psi^{\vee}(U) \in \operatorname{OSp}(\mathscr{S})$, i.e., $D^{-1} U^{*} D=U^{-1}$, we have the following relation:

$$
\begin{aligned}
\left\langle\vec{u}_{-i}^{00}, \vec{u}_{j}^{00}\right\rangle_{B}-\left\langle\vec{u}_{-i}^{10}, \vec{u}_{u_{j}}^{10}\right\rangle_{C} & =(-)^{i} \delta_{i, j}, \\
\left\langle\vec{u}_{-i}^{00}, \vec{u}_{j}^{01}\right\rangle_{B}-\left\langle\vec{u}_{-i}^{10}, \vec{u}_{j}^{11}\right\rangle_{C} & =0, \\
\left\langle\vec{u}_{-i-1}^{01}, \vec{u}_{j}^{00}\right\rangle_{B}+\left\langle\vec{u}_{-i-1}^{11}, \vec{u}_{j}^{10}\right\rangle_{C} & =0, \\
\left\langle\vec{u}_{-i-1}^{01}, \vec{u}_{j}^{01}\right\rangle_{B}+\left\langle\vec{u}_{-i-1}^{11}, \vec{u}_{j}^{11}\right\rangle_{C} & =(-)^{i} \delta_{i, j}, \quad i, j \in \mathbf{Z} .
\end{aligned}
$$

Let $W$ be a supper-wave operator in $\mathscr{E}$ of the OSp-SKP hierarchy and $\Xi$ be the superframe $\Xi=\left.\psi(W) \Xi_{0}\right|_{x=\theta=t=0}$. Put the "check" of $\Xi, \Xi=\left(\left(\vec{\xi}_{i}^{\alpha \beta}\right)_{i<0}\right)_{\alpha, \beta=0,1}$. We note that the entries of $\vec{\xi}_{i}^{\alpha \beta}$ belong to $\mathscr{A}_{\alpha+\beta}$. From $\left.D^{-1} W^{*} D\right|_{x=\theta=t=0}=$ $\left.W^{-1}\right|_{x=\theta=t=0}$, we obtain

$$
\begin{aligned}
& \left\langle\vec{\xi}_{i}^{00}, \vec{\xi}_{j}^{00}\right\rangle_{B}-\left\langle\vec{\xi}_{i}^{10}, \vec{\xi}_{j}^{10}\right\rangle_{C}=0, \\
& \left\langle\vec{\xi}_{i}^{00}, \vec{\xi}_{j}^{01}\right\rangle_{B}-\left\langle\vec{\xi}_{i}^{10}, \vec{\xi}_{j}^{11}\right\rangle_{C}=0, \\
& \left\langle\vec{\xi}_{i}^{01}, \vec{\xi}_{j}^{00}\right\rangle_{B}+\left\langle\vec{\xi}_{i}^{11}, \vec{\xi}_{j}^{10}\right\rangle_{C}=0, \\
& \left\langle\vec{\xi}_{i}^{01}, \vec{\xi}_{j}^{01}\right\rangle_{B}+\left\langle\vec{\xi}_{i}^{11}, \vec{\xi}_{j}^{11}\right\rangle_{C}=0, \quad i, j<0 .
\end{aligned}
$$

Simple computations show that the above condition is invariant under the right action of $S G L\left(\mathbf{N}^{c} ; \mathscr{A}\right)$ on the superframe $\Xi$. We refer to (4.11) as the orthogonality condition. Conversely, let $\Xi$ be a superframe of $U S G M^{\phi}$ satisfying the orthogonality condition. Solve the following Grassmann equation

$$
{ }^{t} \vec{w} \exp \left(\theta \Lambda+x \Lambda^{2}+\sum_{j \equiv 2,3(\bmod 4)} t_{j} \Gamma^{j}\right) \Xi=0 .
$$

The solution to $(4.12){ }^{t} \vec{w}=\left(\ldots, w_{2}, w_{1}, 1,0, \ldots\right), w_{j} \in \mathscr{S}_{\underline{j}}$ determines a super-wave operator $W=\sum_{j=0}^{\infty} w_{j} D^{-j}$ of the SKP hierarchy. Put $W_{0}=\left.W\right|_{t=0}$.

Proposition 4.4. Let $\Xi$ be a superframe satisfying the orthogonality condition and $W$ be the super-wave operator determined by $\Xi$ via (4.12). Then the superframe $\psi^{\vee}\left(W_{0}^{-1}\right) \cdot \check{\Xi}_{0}$ also satisfies the orthogonality condition.

Proof. From $\psi^{\vee}\left(W_{0}\right) \psi^{\vee}\left(W_{0}^{-1}\right)=1$, we obtain the linear equation

$$
{ }^{t} \vec{w}_{0} \psi\left(W_{0}^{-1}\right) \Xi_{0}=0,
$$

where ${ }^{t} \vec{w}_{0}$ is the 0 -th row vector of $\psi\left(W_{0}\right)$. Due to the arguments in Sect. 2, we see that $\psi\left(W_{0}^{-1}\right) \Xi_{0}=\exp \left(\theta \Lambda+x \Lambda^{2}\right) \widetilde{\Xi} \exp \left(-\theta \Lambda_{\mathbf{N}^{c}}-x \Lambda_{\mathbf{N}^{c}}^{2}\right)$, where $\widetilde{\Xi}$ is a superframe of $U S G M^{\phi}$. Then we have

$$
{ }^{t} \vec{w}_{0} \exp \left(\theta \Lambda+x \Lambda^{2}\right) \tilde{\Xi}=0 .
$$

Since (4.12) $\left.\right|_{t=0}$ and (4.14) yield the same solution, $\widetilde{\Xi}=\Xi g$ for some $g \in S G L\left(\mathbf{N}^{c} ; \mathscr{A}\right)$ (see Proposition 2.3.). Hence $\widetilde{\Xi}$ satisfies the orthogonality condition. Moreover observing that $\exp \left(\theta \Lambda+x \Lambda^{2}\right) \in O S p(\mathscr{S})$, we get the conclusion.

Proposition 4.5. Let $U$ be a monic operator in $\mathscr{E}_{0}$ of order 0 . Put $\psi^{\vee}(U)=$ 
$\left(\left(\vec{u}_{i}^{\alpha \beta}\right)_{i \in \mathbf{Z}}\right)_{\alpha, \beta=0,1}$, and suppose that $\vec{u}_{i}^{\alpha \beta}(i<0)$ satisfy the orthogonality condition. Then $\psi^{\vee}(U)$ belongs to $\operatorname{OSp}(\mathscr{S})$, that is $U$ enjoys (4.9).

Proof. Put $A=\left(a_{i j}\right)_{i, j \in \mathbf{Z}}=\psi\left(D^{-1} U^{*} D U\right)$. The entries are given by

$$
\begin{aligned}
a_{2 i, 2 j} & =(-)^{i}\left\{\left\langle\vec{u}_{-i}^{00}, \vec{u}_{j}^{00}\right\rangle_{B}-\left\langle\vec{u}_{-i}^{10}, \vec{u}_{j}^{10}\right\rangle_{C}\right\}, \\
a_{2 i, 2 j+1} & =(-)^{i}\left\{\left\langle\vec{u}_{-i}^{00}, \vec{u}_{j}^{01}\right\rangle_{B}-\left\langle\vec{u}_{-1}^{10}, \vec{u}_{j}^{11}\right\rangle_{C}\right\}, \\
a_{2 i+1,2 j} & =(-)^{i}\left\{\left\langle\vec{u}_{-i-1}^{01}, \vec{u}_{j}^{00}\right\rangle_{B}+\left\langle\vec{u}_{-i-1}^{11}, \vec{u}_{j}^{10}\right\rangle_{C}\right\}, \\
a_{2 i+1,2 j+1} & =(-)^{i}\left\{\left\langle\vec{u}_{-i-1}^{01}, \vec{u}_{j}^{01}\right\rangle_{B}+\left\langle\vec{u}_{-i-1}^{11}, \vec{u}_{j}^{11}\right\rangle_{C}\right\} .
\end{aligned}
$$

Because of the assumption on the orthogonality condition and $D^{-1} U^{*} D U \in$ $\mathscr{E}_{0}$ monic , we can easily see that $a_{i j}=0$ for $i<0, j>0$, and that $a_{i i}=1$ for $i \in \mathbf{Z}$. To show that $D^{-1} U^{*} D U=1$, we have to verify $a_{0,-j}=0$ for $j \geqq 1$, because $D^{-1} U^{*} D U=\sum_{j=0}^{\infty} a_{0,-j} D^{-j}$. We use the induction. Put $U=1+u D^{-1}+$ lower order terms. Then we see that $a_{10}=\left\langle\vec{u}_{-1}^{01}, \vec{u}_{0}^{00}\right\rangle_{B}+\left\langle\vec{u}_{-1}^{11}, \vec{u}_{0}^{10}\right\rangle_{C}=u-u=0$. Since the recursive relation $a_{0, j-1}=(-)^{j-1}\left\{D\left(a_{0 j}\right)-a_{1 j}\right\}$ follows from $D(A)=\Lambda A-A^{\dagger} \Lambda$ (see (2.9)), we get $a_{0,-1}=D\left(a_{00}\right)-a_{10}=0$. By the induction on $j$, we can show that $a_{0,-j}=0$ for $j>0$.

Combining Propositions 4.4 and 4.5, we obtain the following corollary.

Corollary 4.6. Let $\Xi$ be a superframe satisfying the orthogonality condition, and $W(\Xi)$ be the super-wave operator associated with $\Xi$. Then, for the initial value $W_{0}=\left.W(\Xi)\right|_{t=0}, \psi^{\vee}\left(W_{0}\right)$ belongs to $\operatorname{OSp}(\mathscr{S})$.

Let $\pi$ be the set of multi-indices $\alpha=\left(\alpha_{i}\right)_{i=0}^{n}(n \in \mathbf{N})$. We denote $|\alpha|=\sum_{i=0}^{n} \alpha_{i}$ for $\alpha \in \pi$. The void index is denoted by $\phi$. We write $t_{\text {odd }}^{\alpha}=t_{3}^{\alpha_{0}} t_{7}^{\alpha_{1}} \cdots t_{4 n+3}^{\alpha_{n}}$ and $t_{\text {even }}^{\beta}=$ $t_{2}^{\beta_{0}} t_{6}^{\beta_{1}} \cdots t_{4 n+2}^{\beta_{n}}$.

Now we state the main theorem in this section.

Theorem 4.7. Let $W$ be a super-wave operator for the SKP hierarchy associated with the superframe $\Xi \in U S G M^{\phi}$. If $\Xi$ satisfies the orthogonality condition (4.11), then $\left.W\right|_{t_{4 n}=t_{4 n+1}=0}(n \in \mathbf{N})$ is a super-wave operator for the OSp-SKP hierarchy.

Proof. In this proof we set $U=\left.W\right|_{t_{4 n}=t_{4 n+1}=0}$. Expand $U^{*}$ and $U^{-1}$ to the formal power series in $\left(t_{4 n+2}, t_{4 n+3}\right)_{n=0}^{\infty}$ :

$$
U^{*}=\sum_{\alpha, \beta \in \pi} t_{\text {odd }}^{\alpha} t_{\text {even }}^{\beta}\left(U^{*}\right)_{\alpha \beta}, \quad U^{-1}=\sum_{\alpha, \beta \in \pi} t_{\text {odd }}^{\alpha} t_{\mathrm{even}}^{\beta}\left(U^{-1}\right)_{\alpha \beta} .
$$

It is enough to show that

$$
D^{-1}\left(U^{*}\right)_{\alpha \beta} D=(-)^{|\alpha|}\left(U^{-1}\right)_{\alpha \beta} .
$$

We prove (4.20) by the double induction on $|\alpha|$ and $|\beta|$. From Proposition 4.4 and Corollary 4.6, (4.20) holds for $\alpha=\beta=\phi$. Suppose that (4.20) holds for $\alpha=\phi$ and $\beta$ with $|\beta| \leqq m(m \in \mathbf{N})$. Put $\widetilde{\beta}=\left(\widetilde{\beta}_{i}\right)_{i=0}^{n} \in \pi$, where $|\widetilde{\beta}|=m+1$ and $\widetilde{\beta}_{n} \neq 0$. From the equations

$$
\begin{gathered}
D_{4 n+2} U^{*}=-U^{*} B_{4 n+2}^{*}-D^{4 n+2} U^{*} \\
D_{4 n+2} U^{-1}=U^{-1} B_{4 n+2}+D^{4 n+2} U^{-1}
\end{gathered}
$$


which can be deduced from (4.2), we obtain

$$
\tilde{\beta}_{n}\left(U^{*}\right)_{\phi, \tilde{\beta}}=-\sum_{\beta^{\prime}+\beta^{\prime \prime}=\tilde{\beta}-e_{n}}\left(U^{*}\right)_{\phi, \beta^{\prime}}\left(B_{4 n+2}^{*}\right)_{\phi, \beta^{\prime \prime}}-D^{4 n+2}\left(U^{*}\right)_{\phi, \tilde{\beta}-e_{n}},
$$

where $e_{j}=\left(\delta_{i j}\right)_{i=0}^{n} \in \pi$. By (4.20) with $\alpha=\phi$ and $\beta=\beta^{\prime}, \tilde{\beta}-e_{n}$, and by $D^{-1}\left(B_{4 n+2}^{*}\right)_{\phi, \phi} D=$ $-\left(B_{4 n+2}\right)_{\phi, \phi}$, we see that

$$
D^{-1}\left(B_{4 n+2}^{*}\right)_{\phi, \beta^{\prime \prime}} D=-\left(B_{4 n+2}\right)_{\phi, \beta^{\prime \prime}}
$$

Therefore we obtain

$$
\begin{aligned}
\tilde{\beta}_{n} D^{-1}\left(U^{*}\right)_{\phi, \tilde{\beta}} D & =\sum_{\beta^{\prime}+\beta^{\prime \prime}=\tilde{\beta}-e_{n}}\left(U^{-1}\right)_{\phi, \beta^{\prime}}\left(B_{4 n+2}\right)_{\phi, \beta^{\prime \prime}}-\left(U^{-1}\right)_{\phi, \tilde{\beta}-\varepsilon_{n}} D^{4 n+2} \\
& =\widetilde{\beta}_{n}\left(U^{-1}\right)_{\phi, \tilde{\beta}} \quad(\text { by }(4.22)) .
\end{aligned}
$$

Next, suppose that (4.20) holds for $\alpha$ with $|\alpha| \leqq 2 m(m \in \mathbf{N})$ and arbitrary $\beta$. Let $\tilde{\alpha}=\left(\tilde{\alpha}_{i}\right)_{i=0}^{n}$, where $\tilde{\alpha}_{i}=0$ or $1, \tilde{\alpha}=1$ and $|\tilde{\alpha}|=2 m+1$. From the equations

$$
\begin{aligned}
D_{4 n+3}\left(U^{*}\right) & =U^{*} B_{4 n+3}^{*}-D^{4 n+3} U^{*}, \\
D_{4 n+3}\left(U^{-1}\right) & =-U^{-1} B_{4 n+3}+D^{4 n+3} U^{-1},
\end{aligned}
$$

we obtain

$$
\begin{aligned}
\left(U^{*}\right)_{\tilde{\alpha}, \beta}= & \sum_{0 \leqq i \leqq n-1} \tilde{\alpha}_{i} \operatorname{sgn}(i) D_{4 i+4 n+6}\left(U^{*}\right)_{\tilde{\alpha}-e_{i}-e_{n}, \beta} \\
& +\sum_{\substack{\alpha^{\prime}+\alpha^{\prime \prime}=\tilde{\alpha}-e_{n} \\
\beta^{\prime}+\beta^{\prime \prime}=\beta}} \operatorname{sgn}\left(\alpha^{\prime}, \alpha^{\prime \prime}, \alpha^{\prime}\right)\left(U^{*}\right)_{\alpha^{\prime} \beta^{\prime}}\left(B_{4 n+3}^{*}\right)_{\alpha^{\prime \prime} \beta^{\prime \prime}}-D^{4 n+3}\left(U^{*}\right)_{\tilde{\alpha}-e_{n}, \beta} .
\end{aligned}
$$

Here we have defined $\operatorname{sgn}(i)$ and $\operatorname{sgn}\left(\alpha^{\prime}, \alpha^{\prime \prime}, \alpha^{\prime}\right)$ through

$$
\begin{aligned}
t_{4 i+3}^{\tilde{\alpha}_{i} e_{i}} t_{\mathrm{odd}}^{\tilde{\alpha}-\tilde{\alpha}_{i} e_{i}-e_{n}} & =\operatorname{sgn}(i) t_{\mathrm{odd}}^{\tilde{\alpha}-e_{n}}, \\
t_{\text {odd }}^{\alpha^{\prime}} P_{\gamma} t_{\text {odd }}^{\alpha^{\prime \prime}} & =\operatorname{sgn}\left(\alpha^{\prime}, \alpha^{\prime \prime}, \gamma\right) t_{\mathrm{odd}}^{\tilde{\alpha}-\tilde{e}_{n}} P_{\gamma},
\end{aligned}
$$

where $P_{\gamma}$ is an arbitrary monomial of parity $|\gamma| \bmod 2, \gamma \in \pi$. By the induction hypothesis, we see that

$$
\begin{aligned}
D^{-1}\left(U^{*}\right)_{\tilde{\alpha}-e_{n}-\tilde{\alpha}_{i} e_{i}, \beta} D & =-\left(U^{-1}\right)_{\tilde{\alpha}-e_{n}-\tilde{\alpha}_{i} e_{i}, \beta} \text { if } \tilde{\alpha}_{i} \neq 0 \\
D^{-1}\left(U^{*}\right)_{\alpha^{\prime} \beta^{\prime}} D & =(-)^{\left|\alpha^{\prime}\right|}\left(U^{-1}\right)_{\alpha^{\prime} \beta^{\prime}}, \\
D^{-1}\left(U^{*}\right)_{\tilde{\alpha}-e_{n}, \beta} D & =\left(U^{-1}\right)_{\tilde{\alpha}-e_{n}, \beta} .
\end{aligned}
$$

On the other hand, we have

$$
\begin{aligned}
& D^{-1}\left(t_{\text {odd }}^{\alpha^{\prime \prime}} t_{\text {even }}^{\beta^{\prime \prime}}\left(U D^{4 n+3} U^{-1}\right)_{\alpha^{\prime \prime} \beta^{\prime \prime}}\right) * D \\
& =(-)^{\left|\alpha^{\prime \prime}\right|} t_{\text {odd }}^{\alpha^{\prime \prime}} t_{\text {even }}^{\beta^{\prime \prime}} \sum_{\substack{\gamma^{\prime}+\gamma^{\prime \prime}=\alpha^{\prime \prime} \\
\delta^{\prime}+\delta^{\prime \prime}=\beta^{\prime \prime}}} \operatorname{sgn}\left(\gamma^{\prime}, \gamma^{\prime \prime}, \gamma^{\prime}+1\right) D^{-1}\left(\left(U^{-1}\right)^{*}\right)_{\gamma^{\prime} \delta^{\prime}} D^{4 n+3}\left(U^{*}\right)_{\gamma^{\prime \prime} \delta^{\prime \prime}} D .
\end{aligned}
$$

From $D^{-1}\left(\left(U^{-1}\right)^{*}\right)_{\phi \phi} D=(U)_{\phi \phi}$ and the induction hypothesis, we obtain

$$
D^{-1}\left(\left(U^{-1}\right)^{*}\right)_{\gamma^{\prime} \delta^{\prime}} D=(-)^{\left|\gamma^{\prime}\right|}(U)_{\gamma^{\prime} \delta^{\prime}}
$$

and

$$
D^{-1}\left(B_{4 n+3}^{*}\right)_{\alpha^{\prime \prime}, \beta^{\prime \prime}} D=(-)^{\left|\alpha^{\prime \prime}\right|}\left(B_{4 n+3}\right)_{\alpha^{\prime \prime}, \beta^{\prime \prime}} \cdot
$$


Hence we get

$$
\begin{aligned}
D^{-1}\left(U^{*}\right)_{\tilde{\alpha} \beta} D= & \sum_{0 \leqq i \leqq n-1} \tilde{\alpha}_{i} \operatorname{sgn}(i) D_{4 i+4 n+6}\left(U^{-1}\right)_{\tilde{\alpha}-e_{i}-e_{n}, \beta} \\
& +\sum_{\substack{\alpha^{\prime}+\alpha^{\prime \prime}=\tilde{\alpha}-e_{n} \\
\beta^{\prime}+\beta^{\prime \prime}=\beta}} \operatorname{sgn}\left(\alpha^{\prime}, \alpha^{\prime \prime}, \alpha^{\prime}\right)\left(U^{-1}\right)_{\alpha^{\prime} \beta^{\prime}}\left(B_{4 n+3}\right)_{\alpha^{\prime \prime} \beta^{\prime \prime}}-D^{4 n+3}\left(U^{-1}\right)_{\tilde{\alpha}-e_{n}, \beta} .
\end{aligned}
$$

Comparing the right-hand side and the coefficient of $t_{\text {odd }}^{\tilde{\alpha}} t_{\text {even }}^{\beta}$ of $U^{-1}$ in (4.25), we see that

$$
D^{-1}\left(U^{*}\right)_{\tilde{\alpha}, \beta} D=-\left(U^{-1}\right)_{\tilde{\alpha}, \beta} \text {. }
$$

One can show similarly that $D^{-1}\left(U^{*}\right)_{\tilde{\alpha} \beta} D=\left(U^{-1}\right)_{\tilde{\alpha} \beta}$ for the case that $|\alpha|$ is even.

\section{Soliton Solutions to the SKP Hierarchy}

We proceed to the construction of soliton solutions. Let $\alpha_{v}, \beta_{v}, c_{v}$ be even generic elements in $\mathscr{A}$ and $\eta_{v}, \omega_{v}$ be odd ones $(-2 N \leqq v \leqq-1)$. Consider the following condition on a super-wave function:

$$
w(x, \theta, t, \lambda, \xi)=\left(\sum_{j=0}^{2 N} w_{j}(x, \theta, t) D^{-j}\right)(\exp H(x, \theta, t, \lambda, \xi))
$$

and

$$
\begin{aligned}
w\left(x, \theta, t, \alpha_{v}, \eta_{v}\right) & =c_{v} w\left(x, \theta, t, \beta_{v}, \omega_{v}\right) \text { for even } v \\
\left(\left(\hat{D}^{-1}\right)^{*} w\right)\left(x, \theta, t, \alpha_{v}, \eta_{v}\right) & =c_{v}\left(\left(\hat{D}^{-1}\right)^{*} w\right)\left(x, \theta, t, \beta_{v}, \omega_{v}\right) \text { for odd } v .
\end{aligned}
$$

The operator $\left(\hat{D}^{-1}\right)^{*}$ is the formal adjoint operator of $\hat{D}^{-1}$

$$
\left(\hat{D}^{-1}\right)^{*}=-\frac{\partial}{\partial \xi}+\lambda^{-1} \xi
$$

A superanalogue of Cauchy's residue formula reads [8]

$$
\begin{aligned}
& \operatorname{Res}_{\lambda=\alpha}\left\{\Delta(d \lambda / d \xi) \frac{\xi-\eta}{(\lambda-\alpha-\xi \eta)^{n+1}} f(\xi, \eta)\right\}=\frac{1}{n !}\left(D_{\lambda, \xi}^{2 n} f\right)(\alpha, \eta), \\
& \operatorname{Res}_{\lambda=\alpha}\left\{\Delta(d \lambda / d \xi) \frac{1}{(\lambda-\alpha-\xi \eta)^{n+1}} f(\lambda, \xi)\right\}=\frac{1}{n !}\left(D_{\lambda, \xi}^{2 n+1} f\right)(\alpha, \eta),
\end{aligned}
$$

where $D_{\lambda, \xi}=(\partial / \partial \xi)+\xi(\partial / \partial \lambda)$, and $\alpha$ is an even constant, $\eta$ an odd constant. We remark that

$$
\left(\left(\hat{D}^{-1}\right)^{*} w\right)(x, \theta, t, \alpha, \eta)=\operatorname{Res}_{\lambda=\alpha}\left(\Delta(d \lambda / d \xi) W(x, \theta, t, D) D^{-1}(\exp H) \frac{\xi-\eta}{\lambda-\alpha-\xi \eta}\right) .
$$

The condition (5.1) implies the following linear equation:

$$
\begin{gathered}
\left(w_{1}, \ldots, w_{2 N}\right)\left[\left(\phi_{j,-2 v}\right)_{\substack{1 \leqq j \leqq 2 N \\
1 \leqq v \leqq N}} \mid\left(\phi_{j+1,-2 v+1}\right)_{\substack{1 \leqq j \leqq 2 N \\
1 \leqq v \leqq N}}\right] \\
\left.=-\left(\phi_{0,-2 v}\right)_{1 \leqq v \leqq N} \mid\left(\phi_{1,-2 v+1}\right)_{1 \leqq v \leqq N}\right),
\end{gathered}
$$

where 


$$
\phi_{j, v}=\left(D^{-j} \exp H\right)\left(\alpha_{v}, \eta_{v}\right)-c_{v}\left(D^{-j} \exp H\right)\left(\beta_{v}, \omega_{v}\right)
$$

Solving (5.2), one gets an $N$-soliton to the SKP hierarchy. We can rewrite (5.2) into the super-Grassmann equation:

$$
{ }^{t} \vec{w} \Phi \Xi=0
$$

where $\vec{w}=\left(w_{-j}\right)_{j \in \mathbf{Z}}\left(w_{-j}=0\right.$ for $\left.j \geqq 1\right)$,

$$
\begin{aligned}
& \Phi=\exp \left(\theta \Lambda+x \Lambda^{2}+\sum_{n=1}^{\infty} t_{n} \Gamma^{n}\right), \\
& \Lambda=\left(\delta_{\mu+1, v}\right)_{\mu, v \in \mathbf{Z}}, \quad \Gamma=\left((-)^{v} \delta_{\mu+1, v}\right)_{\mu, v \in \mathbf{Z}} \\
& \Xi=\left(\Xi_{\mu, v}\right)_{\mu \in \mathbf{Z}, v \in \mathbf{N}^{c}} \text { with } \\
& \Xi_{\mu, v}=\left[\begin{array}{ll}
\alpha_{v}^{\mu / 2}-c_{v} \beta_{v}{ }^{\mu / 2} & (\mu: \text { even }) \\
-\eta_{v} \alpha_{v}{ }^{(\mu-1) / 2}+c_{v} \omega_{v} \beta_{v}{ }^{(\mu-1) / 2} & (\mu \text { :odd) }
\end{array} \quad \text { for } \quad \begin{array}{l}
-2 N \leqq v<-1, \\
v \text { :even }
\end{array}\right. \\
& \Xi_{\mu, v}=\left[\begin{array}{ll}
-\eta_{v} \alpha_{v}{ }^{-1+\mu / 2}+c_{v} \omega_{v} \beta_{v}{ }^{-1+\mu / 2} & (\mu \text { :even }) \\
\alpha_{n}{ }^{(\mu-1) / 2}-c_{n} \beta_{v}{ }^{(\mu-1) / 2} & (\mu \text { :odd) }
\end{array} \text { for } \quad \begin{array}{l}
v: \text { odd }
\end{array}\right. \\
& \Xi_{\mu, v}=\delta_{\mu, v} \text { for }-\infty<v<-2 N \text {. }
\end{aligned}
$$

Acknowledgement. The authors thank Dr. M. Noumi for stimulating discussions on the basis of the KP hierarchy.

\section{References}

1. Bergvelt, M. J.: A note on super Fock space, preprint, Max-Planck Institut 1988

2. Date, E., Jimbo, M., Kashiwara, M., Miwa, T.: Transformation groups for soliton equations. Proc. RIMS Symp. "Nonlinear Integrable Systems-Classical Theory and Quantum Theory-Miwa, T., Jimbo, M. (eds.), pp. 39-119, Singapore: World Scientific 1983

3. - J. Phys. Soc. Jpn. 40, 3806-3812 (1981)

4. ——: J. Phys. Soc. Jpn. 40, 3813-3818 (1981)

5. — : Physica 4D, 343-365 (1982)

6. ——: Publ. RIMS, Kyoto Univ. 18, 1077-1111 (1982)

7. DeWitt, B.: Supermanifolds. Cambridge: Cambridge Univ. Press 1984

8. Friedan, D., Martinec, E., Shenker, S.: Nucl. Phys. B271, 93-165 (1986)

9. Ikeda, K.: A supersymmetric extension of the Toda lattice hierarchy. Lett. Math. Phys. 14, 321-328 (1987)

10. Kac, V. G., van de Leur, J. W.: Super boson-fermion correspondence. Ann. Inst. Fourier 37, 99-137 (1987)

11. Leites, D. A.: Introduction to the theory of supermanifolds. Russ. Math. Surv. 35:1, 1-64 (1980)

12. Manin, Yu. I., Radul, A. O.: A supersymmetric extension of the Kadomtsev-Petviashvili hierarchy. Commun. Math. Phys. 98, 65-77 (1985)

13. Mulase, M.: Solvability of the super KP equation and a generalization of the Birkhoff decomposition. Invent. Math. 92, 1-46 (1988)

14. Radul, A. O.: Algebra-geometric solutions to the super Kadomtsev-Petviashvili hierarchy, preprint, 1988

15. Sato, M.: Soliton equations and the universal Grassmann manifold, Lecture Note Series, Sophia Univ. No. 18 (1984), (Notes by M. Noumi in Japanese)

16. Sato, M., Sato, Y.: Soliton equations as dynamical systems on an infinite dimensional Grassmann 
manifold. In: Proc. U.S.-Japan Seminar "Nonlinear Partial Differential Equations in Applied Science". Fujita, H., Lax, P. D., Strang, G. (eds.) pp. 259-271. Kinokuniya: North-Holland 1982

17. Ueno, K., Yamada, H.: A supersymmetric extension of nonlinear integrable systems. In: Proc. Conf. "Topological and Geometrical Methods in Field Theory. Westerholm, J., Hietarinta, J. (eds.), pp. 59-72. Singapore: World Scientific 1987

18. -,: Super Kadomtsev-Petviashvili hierarchy and super Grassmann manifold. Lett. Math. Phys. 13, 59-68 (1987)

19. - , Supersymmetric extension of the Kadomtsev-Petviashvili hierarchy and the universal super Grassmann manifold. Adv. Stud. Pure Math. 16, Conformal Field Theory and Solvable Lattice Model (1988), pp. 373-426

20. — hierarchy. To appear in Proc. 16-th Int'l Colloq., "Group Theoretical Methods in Physics"

21. Yamada, H.: Super Grassmann hierarchies-A multicomponent theory-. Hiroshima Math. J. 17, 373-394 (1987)

Communicated by H. Araki

Received November 28, 1988 